Supporting Information (SI) for

\title{
Investigation of the Mechanisms and Kinetics of DBU-Catalyzed PLGA Copolymerization via a Full-Scale Population Balance Analysis
}

\author{
Samruddhi Patil, ${ }^{1}$ Jin Yoo, ${ }^{1}$ and You-Yeon Won ${ }^{1,2, *}$ \\ ${ }^{1}$ Davidson School of Chemical Engineering, Purdue University, West Lafayette, Indiana \\ 47907, USA \\ 2 Purdue University Center for Cancer Research, West Lafayette, Indiana 47906, USA \\ * Corresponding Author (Email: yywon@purdue.edu)
}




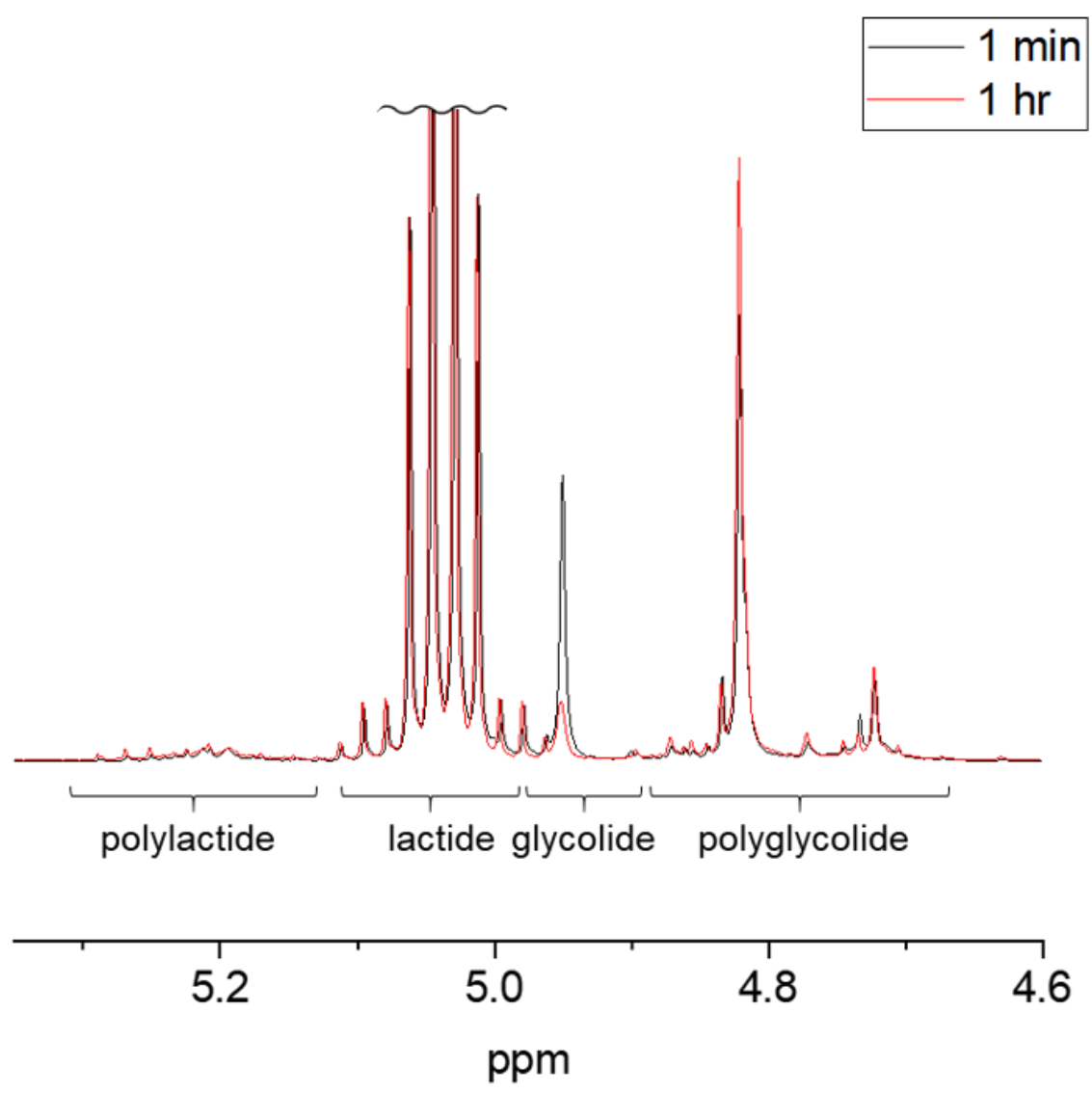

Figure S1. ${ }^{1} \mathrm{H}$ NMR spectra for time samples taken at 1 minute and 1 hour into the DBUcatalyzed LA + GL copolymerization reaction described in Figure 10 of the main text. 
A
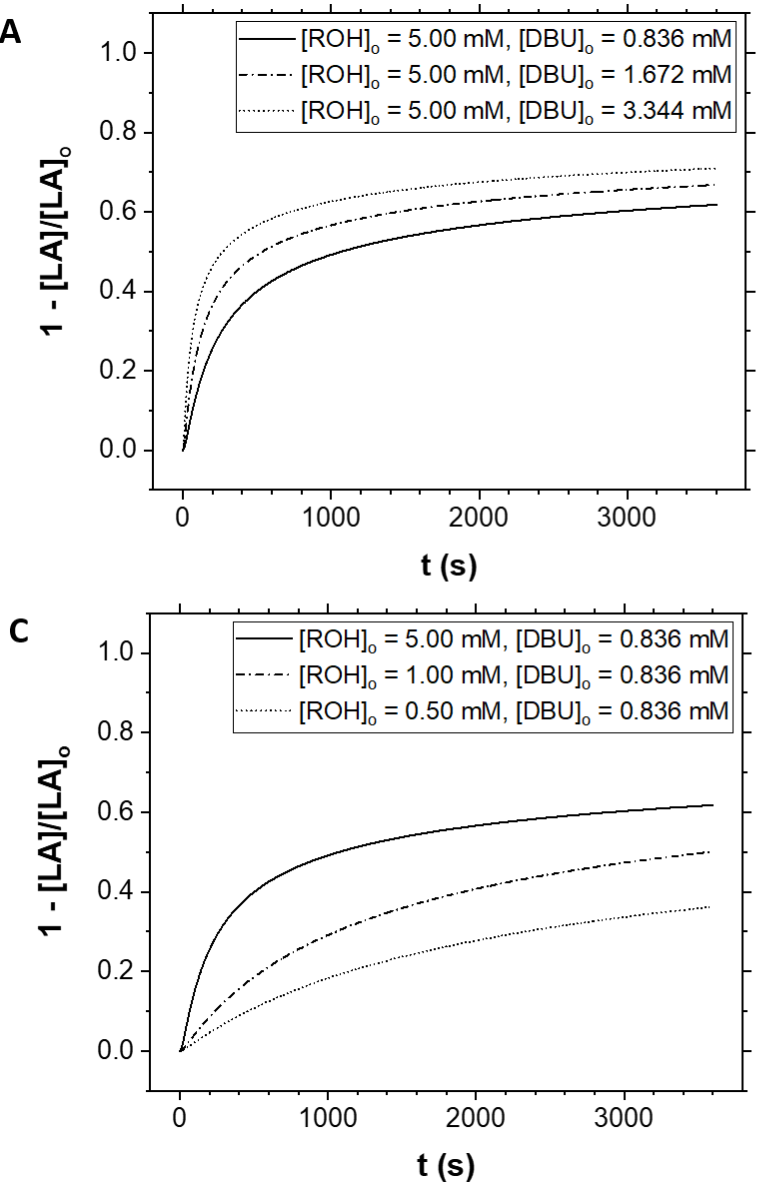

B

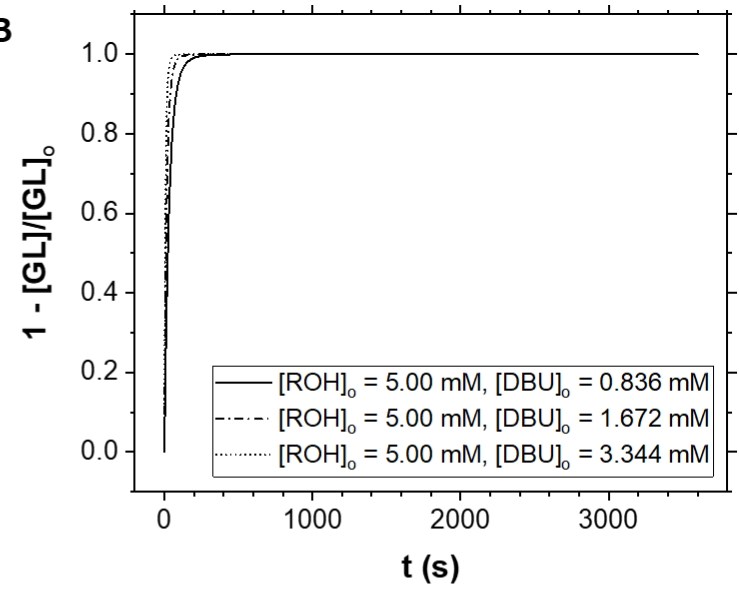

D

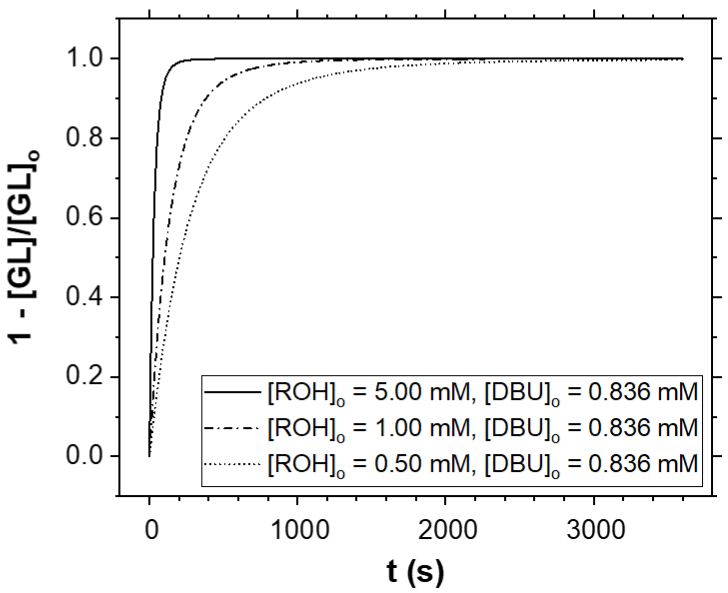

Figure S2. Model predictions of $(\mathbf{A}, \mathbf{C}) \mathrm{LA}$ and $(\mathbf{B}, \mathbf{D})$ GL conversions for the DBUcatalyzed batch copolymerization of LA and GL under different initiator and catalyst concentrations: $[\mathrm{ROH}]_{\mathrm{o}}=0.500,1.00,5.00 \mathrm{mM}$, and $[\mathrm{DBU}]_{\mathrm{o}}=0.836,1.67,3.34 \mathrm{mM}$. The other parameters were the same as in Figure 10: $[\mathrm{LA}]_{\mathrm{o}}=60.8 \mathrm{mM},[\mathrm{GL}]_{\mathrm{o}}=15.0 \mathrm{mM}$, solvent $=\mathrm{CDCl}_{3}, \mathrm{~T}=25{ }^{\circ} \mathrm{C}$. The predictions were calculated based on the kinetic model described in Sections S1 - S5 of the SI using the rate constant values listed in Table 3. 


\section{S1. Kinetic balance equations for the DBU-catalyzed copolymerization of LA and GL in a batch reactor}

\section{Notations :}

$D=\mathrm{DBU}$

$M_{i}=\operatorname{monomer}(i=1$ for LA and 2 for GL)

$I=$ initiator $(\mathrm{ROH})$

$[D-I]=$ DBU-initiator complex

$D_{i, 1}=\mathrm{DBU}$-monomer $i$ complex

$D_{i, n}^{*}=$ growing chain of length $n$ with the initiator fragment (-OR) on one end and monomer $i$ $+D B U^{+}$on the other

$C_{i, n}=$ cyclic chain of length $n$ formed from a chain with a monomer $i$ end group

$K A_{i, n}=$ dormant chain of length $n$ terminated by a KA on one end and monomer $i+-\mathrm{OH}$ on the other

$K A_{i, n}^{*}=$ growing chain of length $n$ terminated by a KA on one end and monomer $i+D B U^{+}$on the other

$K=$ acidic contaminant

Generally, in the $\left[S_{i, n}^{*}\right]$ notation, $S$ denotes species type, * state of end group, $i$ last type of monomer added to the species, $n$ length of the species, and [] molar concentration.

(S.1. 1) $\frac{d[D]}{d t}=-k_{a}^{1}[D][I]+k_{d}^{1}[[D-I]]-k_{a 1}^{1}[D]\left[\sum_{n=1}^{\infty}\left[R_{1, n}\right]+\sum_{m=1}^{\infty}\left[K A_{1, m}\right]+\right.$ $\left.\sum_{n=1}^{\infty}\left[R_{2, n}\right]+\sum_{m=1}^{\infty}\left[K A_{2, m}\right]\right]+k_{d 1}^{1}\left[\sum_{n=1}^{\infty}\left[D_{1, n}^{*}\right]+\sum_{m=1}^{\infty}\left[K A_{1, m}^{*}\right]+\sum_{n=1}^{\infty}\left[D_{2, n}^{*}\right]+\right.$ $\left.\sum_{m=1}^{\infty}\left[K A_{2, m}^{*}\right]\right]-k_{a 1}^{2}[D]\left[M_{1}\right]-k_{a 2}^{2}[D]\left[M_{2}\right]+k_{d 1}^{2}\left[K A_{1,1}\right]+k_{d 2}^{2}\left[K A_{2,1}\right]-k_{a 1}^{1}\left[K A_{1,1}\right][D]+$ $k_{d 1}^{1}\left[K A_{1,1}^{*}\right]-k_{a 2}^{1}\left[K A_{2,1}\right][D]+k_{d 2}^{1}\left[K A_{2,1}^{*}\right]-k_{t}^{3}[D][K]+k_{a c 1}^{1}\left[\sum_{m=1}^{\infty}\left[K A_{1, m}^{*}\right]+\right.$ $\left.\sum_{m=1}^{\infty}\left[K A_{1, m}\right]\right] *\left[[I]+\sum_{m=1}^{\infty}\left[K A_{1, m}\right]+\sum_{m=1}^{\infty}\left[R_{1, m}\right]+\sum_{m=1}^{\infty}\left[K A_{2, m}\right]+\sum_{m=1}^{\infty}\left[R_{2, m}\right]\right]+$ $k_{a c 1}^{1}\left[K A_{1, m}\right]\left[\sum_{n=1}^{\infty}\left[K A_{1, n}\right]+\sum_{n=1}^{\infty}\left[K A_{2, n}\right]\right]+k_{a c 2}^{1}\left[\sum_{m=1}^{\infty}\left[K A_{2, m}^{*}\right]+\sum_{m=1}^{\infty}\left[K A_{2, m}\right]\right] *$ $\left[[I]+\sum_{m=1}^{\infty}\left[K A_{1, m}\right]+\sum_{m=1}^{\infty}\left[R_{1, m}\right]+\sum_{m=1}^{\infty}\left[K A_{2, m}\right]+\sum_{m=1}^{\infty}\left[R_{2, m}\right]\right]+$ $k_{a c 2}^{1}\left[K A_{2, m}\right]\left[\sum_{n=1}^{\infty}\left[K A_{1, n}\right]+\sum_{n=1}^{\infty}\left[K A_{2, n}\right]\right]+k_{a c 1}^{2} \sum_{m=1}^{\infty}\left[K A_{1, m}\right]+k_{a c 2}^{2} \sum_{m=1}^{\infty}\left[K A_{2, m}\right]-$ $k_{t}^{4}[D][K]-k_{t}^{5}[D]\left[D^{+}\right]$

(S.1. 2.1) $\frac{d\left[M_{1}\right]}{d t}=-k_{i 1}[[D-I]]\left[M_{1}\right]-k_{p(1,1)}^{1}\left[M_{1}\right]\left[\sum_{n=1}^{\infty}\left[D_{1, n}^{*}\right]+\sum_{m=1}^{\infty}\left[K A_{1, m}^{*}\right]\right]-$ $k_{p(2,1)}^{1}\left[M_{1}\right]\left[\sum_{n=1}^{\infty}\left[D_{2, n}^{*}\right]+\sum_{m=1}^{\infty}\left[K A_{2, m}^{*}\right]\right]-k_{a 1}^{2}[D]\left[M_{1}\right]+k_{d 1}^{2}\left[K A_{1,1}\right]+k_{a c 1}^{2}\left[K A_{1,1}\right]$ $\left(\right.$ S.1. 2.2) $\frac{d\left[M_{2}\right]}{d t}=-k_{i 2}[D-I]\left[M_{2}\right]-k_{p(1,2)}^{1}\left[M_{2}\right]\left[\sum_{n=1}^{\infty}\left[D_{1, n}^{*}\right]+\sum_{m=1}^{\infty}\left[K A_{1, m}^{*}\right]\right]-$ $k_{p(2,2)}^{1}\left[M_{2}\right]\left[\sum_{n=1}^{\infty}\left[D_{2, n}^{*}\right]+\sum_{m=1}^{\infty}\left[K A_{2, m}^{*}\right]\right]-k_{a 2}^{2}[D]\left[M_{2}\right]+k_{d 2}^{2}\left[K A_{2,1}\right]+k_{a c 2}^{2}\left[K A_{2,1}\right]$ 
(S.1.3) $\frac{d[I]}{d t}=-k_{a}^{1}[I][D]+k_{d}^{1}[[D-I]]-k_{a c 1}^{1}[I]\left[\sum_{m=1}^{\infty}\left[K A_{1, m}^{*}\right]+\right.$ $\left.\sum_{m=1}^{\infty}\left[K A_{1, m}\right]\right]+k_{a c 2}^{1}[I]\left[\sum_{m=1}^{\infty}\left[K A_{2, m}^{*}\right]+\sum_{m=1}^{\infty}\left[K A_{2, m}\right]\right]$

(S. 1. 4) $\frac{d[D-I]}{d t}=k_{a}^{1}[I][D]-k_{d}^{1}[[D-I]]-k_{i 1}\left[M_{1}\right][D-I]-k_{i 2}\left[M_{2}\right][D-I]$

(S. 1. 5) $\frac{d\left[D^{d}\right]}{d t}=k_{t}^{1}[K]\left[\sum_{m=1}^{\infty}\left[D_{1, m}^{*}\right]+\sum_{m=1}^{\infty}\left[K A_{1, m}^{*}\right]+\sum_{m=1}^{\infty}\left[D_{2, m}^{*}\right]+\sum_{m=1}^{\infty}\left[K A_{2, m}^{*}\right]\right]+$ $k_{t}^{2}\left[\sum_{m=1}^{\infty}\left[D_{1, m}^{*}\right]+\sum_{m=1}^{\infty}\left[K A_{1, m}^{*}\right]+\sum_{m=1}^{\infty}\left[D_{2, m}^{*}\right]+\sum_{m=1}^{\infty}\left[K A_{2, m}^{*}\right]\right]+$ $k_{d}\left[\sum_{n=1}^{\infty}\left[D_{1, n}^{*}\right]\right]\left[\sum_{m=1}^{\infty}\left[D_{1, m}^{*}\right]+\sum_{m=1}^{\infty}\left[K A_{1, m}^{*}\right]+\sum_{m=1}^{\infty}\left[D_{2, m}^{*}\right]+\sum_{m=1}^{\infty}\left[K A_{2, m}^{*}\right]\right]+$ $k_{d}\left[\sum_{n=1}^{\infty}\left[D_{2, n}^{*}\right]\right]\left[\sum_{m=1}^{\infty}\left[D_{1, m}^{*}\right]+\sum_{m=1}^{\infty}\left[K A_{1, m}^{*}\right]+\sum_{m=1}^{\infty}\left[D_{2, m}^{*}\right]+\sum_{m=1}^{\infty}\left[K A_{2, m}^{*}\right]\right]+$ $k_{d}\left[\sum_{n=1}^{\infty}\left[K A_{1, n}^{*}\right]\right]\left[\sum_{m=1}^{\infty}\left[D_{1, m}^{*}\right]+\sum_{m=1}^{\infty}\left[K A_{1, m}^{*}\right]+\sum_{m=1}^{\infty}\left[D_{2, m}^{*}\right]+\sum_{m=1}^{\infty}\left[K A_{2, m}^{*}\right]\right]+$ $k_{d}\left[\sum_{n=1}^{\infty}\left[K A_{2, n}^{*}\right]\right]\left[\sum_{m=1}^{\infty}\left[D_{1, m}^{*}\right]+\sum_{m=1}^{\infty}\left[K A_{1, m}^{*}\right]+\sum_{m=1}^{\infty}\left[D_{2, m}^{*}\right]+\sum_{m=1}^{\infty}\left[K A_{2, m}^{*}\right]\right]+$ $k_{t}^{3}[K][D]+k_{t}^{5}[D]\left[D^{+}\right]$

(S.1.6) $\frac{d[K]}{d t}=-k_{t}^{1}[K]\left[\sum_{m=1}^{\infty}\left[D_{1, m}^{*}\right]+\sum_{m=1}^{\infty}\left[K A_{1, m}^{*}\right]+\sum_{m=1}^{\infty}\left[D_{2, m}^{*}\right]+\sum_{m=1}^{\infty}\left[K A_{2, m}^{*}\right]\right]-$ $k_{t}^{3}[K][D]-k_{t}^{4}[D][K]$

(S.1. 7.1) $n=1: \frac{d\left[D_{1,1}^{*}\right]}{d t}=k_{i 1}^{1}\left[M_{1}\right][[D-I]]+k_{a 1}^{1}[D]\left[R_{1,1}\right]-k_{d 1}^{1}\left[D_{1,1}^{*}\right]-$ $k_{p(1,1)}^{1}\left[M_{1}\right]\left[D_{1,1}^{*}\right]-k_{p(1,2)}^{1}\left[M_{2}\right]\left[D_{1,1}^{*}\right]-k_{t}^{1}[K]\left[D_{1,1}^{*}\right]-k_{t}^{2}\left[D_{1,1}^{*}\right]-k_{d}\left[D_{1,1}^{*}\right]\left[\sum_{m=1}^{\infty}\left[D_{1, m}^{*}\right]+\right.$ $\left.\sum_{m=1}^{\infty}\left[K A_{1, m}^{*}\right]+\sum_{m=1}^{\infty}\left[D_{2, m}^{*}\right]+\sum_{m=1}^{\infty}\left[K A_{2, m}^{*}\right]\right]+k_{a c 1}^{1}[I]\left[K A_{1,1}^{*}\right]$

$\left(\right.$ S.1. 7.2) $n=1: \frac{d\left[D_{2,1}^{*}\right]}{d t}=k_{i 2}^{1}\left[M_{2}\right][[D-I]]+k_{a 2}^{1}[D]\left[R_{2,1}\right]-k_{d 2}^{1}\left[D_{2,1}^{*}\right]-$ $k_{p(2,1)}^{1}\left[M_{1}\right]\left[D_{2,1}^{*}\right]-k_{p(2,2)}^{1}\left[M_{2}\right]\left[D_{2,1}^{*}\right]-k_{t}^{1}[K]\left[D_{2,1}^{*}\right]-k_{t}^{2}\left[D_{2,1}^{*}\right]-k_{d}\left[D_{2,1}^{*}\right]\left[\sum_{m=1}^{\infty}\left[D_{1, m}^{*}\right]+\right.$ $\left.\sum_{m=1}^{\infty}\left[K A_{1, m}^{*}\right]+\sum_{m=1}^{\infty}\left[D_{2, m}^{*}\right]+\sum_{m=1}^{\infty}\left[K A_{2, m}^{*}\right]\right]+k_{a c 2}^{1}[I]\left[K A_{2,1}^{*}\right]$

(S.1. 8. 1) $n>1: \frac{d\left[D_{1, n}^{*}\right]}{d t}=k_{a 1}^{1}[D]\left[R_{1, n}\right]-k_{d 1}^{1}\left[D_{1, n}^{*}\right]-k_{p(1,1)}^{1}\left[M_{1}\right]\left[D_{1, n}^{*}\right]-$ $k_{p(1,2)}^{1}\left[M_{2}\right]\left[D_{1, n}^{*}\right]+k_{p(1,1)}^{1}\left[M_{1}\right]\left[D_{1, n-1}^{*}\right]+k_{p(2,1)}^{1}\left[M_{1}\right]\left[D_{2, n-1}^{*}\right]-k_{t}^{1}[K]\left[D_{1, n}^{*}\right]-k_{t}^{2}\left[D_{1, n}^{*}\right]-$ $k_{d}\left[D_{1, n}^{*}\right]\left[\sum_{m=1}^{\infty}\left[D_{1, m}^{*}\right]+\sum_{m=1}^{\infty}\left[K A_{1, m}^{*}\right]+\sum_{m=1}^{\infty}\left[D_{2, m}^{*}\right]+\sum_{m=1}^{\infty}\left[K A_{2, m}^{*}\right]\right]+k_{a c 1}^{1}[I]\left[K A_{1, n}^{*}\right]+$ $k_{a c 1}^{1}\left(\sum_{i=1}^{n-1}\left[K A_{1, i}^{*}\right]\left[R_{1, n-i}\right]\right)+k_{a c 1}^{1}\left(\sum_{i=1}^{n-1}\left[K A_{1, i}^{*}\right]\left[R_{2, n-i}\right]\right)$

(S.1. 8.2) $n>1$ : $\frac{d\left[D_{2, n}^{*}\right]}{d t}=k_{a 2}^{1}[D]\left[R_{2, n}\right]-k_{d 2}^{1}\left[D_{2, n}^{*}\right]-k_{p(2,1)}^{1}\left[M_{1}\right]\left[D_{2, n}^{*}\right]-$ $k_{p(2,2)}^{1}\left[M_{2}\right]\left[D_{2, n}^{*}\right]+k_{p(1,2)}^{1}\left[M_{2}\right]\left[D_{1, n-1}^{*}\right]+k_{p(2,2)}^{1}\left[M_{2}\right]\left[D_{2, n-1}^{*}\right]-k_{t}^{1}[K]\left[D_{2, n}^{*}\right]-k_{t}^{2}\left[D_{2, n}^{*}\right]-$ $k_{d}\left[D_{2, n}^{*}\right]\left[\sum_{m=1}^{\infty}\left[D_{1, m}^{*}\right]+\sum_{m=1}^{\infty}\left[K A_{1, m}^{*}\right]+\sum_{m=1}^{\infty}\left[D_{2, m}^{*}\right]+\sum_{m=1}^{\infty}\left[K A_{2, m}^{*}\right]\right]+k_{a c 2}^{1}[I]\left[K A_{2, n}^{*}\right]+$ $k_{a c 2}^{1}\left(\sum_{i=1}^{n-1}\left[K A_{2, i}^{*}\right]\left[R_{1, n-i}\right]\right)+k_{a c 2}^{1}\left(\sum_{i=1}^{n-1}\left[K A_{2, i}^{*}\right]\left[R_{2, n-i}\right]\right)$

(S.1. 9.1) $n=1: \frac{d\left[R_{1,1}\right]}{d t}=-k_{a 1}^{1}[D]\left[R_{1,1}\right]+k_{d 1}^{1}\left[D_{1,1}^{*}\right]+k_{t}^{1}[K]\left[D_{1,1}^{*}\right]+k_{t}^{2}\left[D_{1,1}^{*}\right]+$ $k_{d}\left[D_{1,1}^{*}\right]\left[\sum_{m=1}^{\infty}\left[D_{1, m}^{*}\right]+\sum_{m=1}^{\infty}\left[K A_{1, m}^{*}\right]+\sum_{m=1}^{\infty}\left[D_{2, m}^{*}\right]+\sum_{m=1}^{\infty}\left[K A_{2, m}^{*}\right]\right]+k_{a c 1}^{1}[I]\left[K A_{1,1}\right]-$ $k_{a c 1}^{1}\left[R_{1,1}\right] *\left(\sum_{m=1}^{\infty}\left[K A_{1, m}^{*}\right]+\sum_{m=1}^{\infty}\left[K A_{1, m}\right]+\sum_{m=1}^{\infty}\left[K A_{2, m}^{*}\right]+\sum_{m=1}^{\infty}\left[K A_{2, m}\right]\right)$ 
$\left(\boldsymbol{S . 1 . 9 . 2 )} n=1: \frac{d\left[R_{2,1}\right]}{d t}=-k_{a 2}^{1}[D]\left[R_{2,1}\right]+k_{d 2}^{1}\left[D_{2,1}^{*}\right]+k_{t}^{1}[K]\left[D_{2,1}^{*}\right]+k_{t}^{2}\left[D_{2,1}^{*}\right]+\right.$ $k_{d}\left[D_{2,1}^{*}\right]\left[\sum_{m=1}^{\infty}\left[D_{1, m}^{*}\right]+\sum_{m=1}^{\infty}\left[K A_{1, m}^{*}\right]+\sum_{m=1}^{\infty}\left[D_{2, m}^{*}\right]+\sum_{m=1}^{\infty}\left[K A_{2, m}^{*}\right]\right]+k_{a c 2}^{1}[I]\left[K A_{2,1}\right]-$ $k_{a c 2}^{1}\left[R_{2,1}\right] *\left(\sum_{m=1}^{\infty}\left[K A_{1, m}^{*}\right]+\sum_{m=1}^{\infty}\left[K A_{1, m}\right]+\sum_{m=1}^{\infty}\left[K A_{2, m}^{*}\right]+\sum_{m=1}^{\infty}\left[K A_{2, m}\right]\right)$

(S.1. 10.1) $n>1: \frac{d\left[R_{1, n}\right]}{d t}=-k_{a 1}^{1}[D]\left[R_{1, n}\right]+k_{d 1}^{1}\left[D_{1, n}^{*}\right]+k_{t}^{1}[K]\left[D_{1, n}^{*}\right]+k_{t}^{2}\left[D_{1, n}^{*}\right]+$ $k_{d}\left[D_{1, n}^{*}\right]\left[\sum_{m=1}^{\infty}\left[D_{1, m}^{*}\right]+\sum_{m=1}^{\infty}\left[K A_{1, m}^{*}\right]+\sum_{m=1}^{\infty}\left[D_{2, m}^{*}\right]+\sum_{m=1}^{\infty}\left[K A_{2, m}^{*}\right]\right]+k_{a c 1}^{1}[I]\left[K A_{1, n}\right]-$ $k_{a c 1}^{1}\left[R_{1, n}\right] *\left(\sum_{m=1}^{\infty}\left[K A_{1, m}^{*}\right]+\sum_{m=1}^{\infty}\left[K A_{1, m}\right]+\sum_{m=1}^{\infty}\left[K A_{2, m}^{*}\right]+\sum_{m=1}^{\infty}\left[K A_{2, m}\right]\right)+$ $k_{a c 1}^{1}\left(\sum_{i=1}^{n-1}\left[K A_{1, i}\right]\left[R_{1, n-i}\right]\right)+k_{a c 1}^{1}\left(\sum_{i=1}^{n-1}\left[K A_{1, i}\right]\left[R_{2, n-i}\right]\right)$

(S.1. 10.2) $n>1$ : $\frac{d\left[R_{2, n}\right]}{d t}=-k_{a 2}^{1}[D]\left[R_{2, n}\right]+k_{d 2}^{1}\left[D_{2, n}^{*}\right]+k_{t}^{1}[K]\left[D_{2, n}^{*}\right]+k_{t}^{2}\left[D_{2, n}^{*}\right]+$ $k_{d}\left[D_{2, n}^{*}\right]\left[\sum_{m=1}^{\infty}\left[D_{1, m}^{*}\right]+\sum_{m=1}^{\infty}\left[K A_{1, m}^{*}\right]+\sum_{m=1}^{\infty}\left[D_{2, m}^{*}\right]+\sum_{m=1}^{\infty}\left[K A_{2, m}^{*}\right]\right]+k_{a c 2}^{1}[I]\left[K A_{2, n}\right]-$ $k_{a c 2}^{1}\left[R_{2, n}\right] *\left(\sum_{m=1}^{\infty}\left[K A_{1, m}^{*}\right]+\sum_{m=1}^{\infty}\left[K A_{1, m}\right]+\sum_{m=1}^{\infty}\left[K A_{2, m}^{*}\right]+\sum_{m=1}^{\infty}\left[K A_{2, m}\right]\right)+$ $k_{a c 2}^{1}\left(\sum_{i=1}^{n-1}\left[K A_{2, i}\right]\left[R_{1, n-i}\right]\right)+k_{a c 2}^{1}\left(\sum_{i=1}^{n-1}\left[K A_{2, i}\right]\left[R_{2, n-i}\right]\right)$

(S.1. 11.1) $m=1: \frac{d\left[K A_{1,1}^{*}\right]}{d t}=-k_{p(1,1)}^{1}\left[M_{1}\right]\left[K A_{1,1}^{*}\right]-k_{p(1,2)}^{1}\left[M_{2}\right]\left[K A_{1,1}^{*}\right]-$ $k_{t}^{1}\left[K A_{1,1}^{*}\right][K]+2 k_{a 1}^{1}[D]\left[K A_{1,1}\right]-2 k_{d 1}^{1}\left[K A_{1,1}^{*}\right]-k_{t}^{2}\left[K A_{1,1}^{*}\right]-k_{d}\left[K A_{1,1}^{*}\right]\left[\sum_{m=1}^{\infty}\left[D_{1, m}^{*}\right]+\right.$ $\left.\sum_{m=1}^{\infty}\left[K A_{1, m}^{*}\right]+\sum_{m=1}^{\infty}\left[D_{2, m}^{*}\right]+\sum_{m=1}^{\infty}\left[K A_{2, m}^{*}\right]\right]-k_{a c 1}^{1}\left[K A_{1,1}^{*}\right] *\left[[I]+\sum_{n=1}^{\infty}\left[K A_{1, n}\right]+\right.$ $\left.\sum_{n=1}^{\infty}\left[R_{1, n}\right]+\sum_{n=1}^{\infty}\left[K A_{2, n}\right]+\sum_{n=1}^{\infty}\left[R_{2, n}\right]\right]$

(S.1. 11.2) $m=1: \frac{d\left[K A_{2,1}^{*}\right]}{d t}=-k_{p(2,1)}^{1}\left[M_{1}\right]\left[K A_{2,1}^{*}\right]-k_{p(2,2)}^{1}\left[M_{2}\right]\left[K A_{2,1}^{*}\right]-$ $k_{t}^{1}\left[K A_{2,1}^{*}\right][K]+2 k_{a 2}^{1}[D]\left[K A_{2,1}\right]-2 k_{d 2}^{1}\left[K A_{2,1}^{*}\right]-k_{t}^{2}\left[K A_{2,1}^{*}\right]-k_{d}\left[K A_{2,1}^{*}\right]\left[\sum_{m=1}^{\infty}\left[D_{1, m}^{*}\right]+\right.$ $\left.\sum_{m=1}^{\infty}\left[K A_{1, m}^{*}\right]+\sum_{m=1}^{\infty}\left[D_{2, m}^{*}\right]+\sum_{m=1}^{\infty}\left[K A_{2, m}^{*}\right]\right]-k_{a c 2}^{1}\left[K A_{2,1}^{*}\right] *\left[[I]+\sum_{n=1}^{\infty}\left[K A_{1, n}\right]+\right.$ $\left.\sum_{n=1}^{\infty}\left[R_{1, n}\right]+\sum_{n=1}^{\infty}\left[K A_{2, n}\right]+\sum_{n=1}^{\infty}\left[R_{2, n}\right]\right]$

(S.1. 12.1) $m>1: \frac{d\left[K A_{1, m}^{*}\right]}{d t}=k_{p(1,1)}^{1}\left[M_{1}\right]\left[K A_{1, m-1}^{*}\right]+k_{p(2,1)}^{1}\left[M_{1}\right]\left[K A_{2, m-1}^{*}\right]-$ $k_{p(1,1)}^{1}\left[M_{1}\right]\left[K A_{1, m}^{*}\right]-k_{p(1,2)}^{1}\left[M_{2}\right]\left[K A_{1, m}^{*}\right]-k_{t}^{1}\left[K A_{1, m}^{*}\right][K]+k_{a 1}^{1}[D]\left[K A_{1, m}\right]-k_{d 1}^{1}\left[K A_{1, m}^{*}\right]$ $-k_{t}^{2}\left[K A_{1, m}^{*}\right]-k_{d}\left[K A_{1, m}^{*}\right]\left[\sum_{m=1}^{\infty}\left[D_{1, m}^{*}\right]+\sum_{m=1}^{\infty}\left[K A_{1, m}^{*}\right]+\sum_{m=1}^{\infty}\left[D_{2, m}^{*}\right]+\sum_{m=1}^{\infty}\left[K A_{2, m}^{*}\right]\right]-$ $k_{a c 1}^{1}\left[K A_{1, m}^{*}\right] *\left[[I]+\sum_{n=1}^{\infty}\left[K A_{1, n}\right]+\sum_{n=1}^{\infty}\left[R_{1, n}\right]+\sum_{n=1}^{\infty}\left[K A_{2, n}\right]+\sum_{n=1}^{\infty}\left[R_{2, n}\right]\right]+$ $k_{a c 1}^{1}\left(\sum_{i=1}^{m-1}\left[K A_{1, i}^{*}\right]\left[K A_{1, m-i}\right]\right)+k_{a c 1}^{1}\left(\sum_{i=1}^{m-1}\left[K A_{1, i}^{*}\right]\left[K A_{2, m-i}\right]\right)$

(S.1. 12.2) $m>1$ : $\frac{d\left[K A_{2, m}^{*}\right]}{d t}=k_{p(1,2)}^{1}\left[M_{2}\right]\left[K A_{1, m-1}^{*}\right]+k_{p(2,2)}^{1}\left[M_{2}\right]\left[K A_{2, m-1}^{*}\right]-$ $k_{p(2,1)}^{1}\left[M_{1}\right]\left[K A_{2, m}^{*}\right]-k_{p(2,2)}^{1}\left[M_{2}\right]\left[K A_{2, m}^{*}\right]-k_{t}^{1}\left[K A_{2, m}^{*}\right][K]+k_{a 2}^{1}[D]\left[K A_{2, m}\right]-k_{d 2}^{1}\left[K A_{2, m}^{*}\right]$ $-k_{t}^{2}\left[K A_{2, m}^{*}\right]-k_{d}\left[K A_{2, m}^{*}\right]\left[\sum_{m=1}^{\infty}\left[D_{1, m}^{*}\right]+\sum_{m=1}^{\infty}\left[K A_{1, m}^{*}\right]+\sum_{m=1}^{\infty}\left[D_{2, m}^{*}\right]+\sum_{m=1}^{\infty}\left[K A_{2, m}^{*}\right]\right]-$ $k_{a c 2}^{1}\left[K A_{2, m}^{*}\right] *\left[[I]+\sum_{n=1}^{\infty}\left[K A_{1, n}\right]+\sum_{n=1}^{\infty}\left[R_{1, n}\right]+\sum_{n=1}^{\infty}\left[K A_{2, n}\right]+\sum_{n=1}^{\infty}\left[R_{2, n}\right]\right]+$ $k_{a c 2}^{1}\left(\sum_{i=1}^{m-1}\left[K A_{2, i}^{*}\right]\left[K A_{1, m-i}\right]\right)+k_{a c 2}^{1}\left(\sum_{i=1}^{m-1}\left[K A_{2, i}^{*}\right]\left[K A_{2, m-i}\right]\right)$ 
(S.1. 13.1) $m=1: \frac{d\left[K A_{1,1}\right]}{d t}=k_{a 1}^{2}\left[M_{1}\right][D]-k_{d 1}^{2}\left[K A_{1,1}\right]-2 k_{a 1}^{1}[D]\left[K A_{1,1}\right]+$ $2 k_{d 1}^{1}\left[K A_{1,1}^{*}\right]+k_{t}^{1}[K]\left[K A_{1,1}^{*}\right]+k_{t}^{2}\left[K A_{1,1}^{*}\right]+k_{d}\left[K A_{1,1}^{*}\right]\left[\sum_{m=1}^{\infty}\left[D_{1, m}^{*}\right]+\sum_{m=1}^{\infty}\left[K A_{1, m}^{*}\right]+\right.$ $\left.\sum_{m=1}^{\infty}\left[D_{2, m}^{*}\right]+\sum_{m=1}^{\infty}\left[K A_{2, m}^{*}\right]\right]-k_{a c 1}^{1}\left[K A_{1,1}\right] *\left[[I]+\sum_{n=1}^{\infty}\left[K A_{1, n}^{*}\right]+\sum_{n=1}^{\infty}\left[R_{1, n}\right]+\right.$ $\left.\sum_{n=1}^{\infty}\left[K A_{2, n}^{*}\right]+\sum_{n=1}^{\infty}\left[R_{2, n}\right]\right]-2 k_{a c 1}^{1}\left[K A_{1,1}\right]\left[\sum_{n=1}^{\infty}\left[K A_{1, n}\right]+\sum_{n=1}^{\infty}\left[K A_{2, n}\right]\right]-k_{a c 1}^{2}\left[K A_{1,1}\right]$

(S.1. 13.2) $m=1: \frac{d\left[K A_{2,1}\right]}{d t}=k_{a 2}^{2}\left[M_{2}\right][D]-k_{d 2}^{2}\left[D_{2,1}\right]-2 k_{a 2}^{1}[D]\left[K A_{2,1}\right]+$ $2 k_{d 2}^{1}\left[K A_{2,1}^{*}\right]+k_{t}^{1}[K]\left[K A_{2,1}^{*}\right]+k_{t}^{2}\left[K A_{2,1}^{*}\right]+k_{d}\left[K A_{2,1}^{*}\right]\left[\sum_{m=1}^{\infty}\left[D_{1, m}^{*}\right]+\sum_{m=1}^{\infty}\left[K A_{1, m}^{*}\right]+\right.$ $\left.\sum_{m=1}^{\infty}\left[D_{2, m}^{*}\right]+\sum_{m=1}^{\infty}\left[K A_{2, m}^{*}\right]\right]-k_{a c 2}^{1}\left[K A_{2,1}\right] *\left[[I]+\sum_{n=1}^{\infty}\left[K A_{1, n}^{*}\right]+\sum_{n=1}^{\infty}\left[R_{1, n}\right]+\right.$ $\left.\sum_{n=1}^{\infty}\left[K A_{2, n}^{*}\right]+\sum_{n=1}^{\infty}\left[R_{2, n}\right]\right]-2 k_{a c 2}^{1}\left[K A_{2,1}\right]\left[\sum_{n=1}^{\infty}\left[K A_{1, n}\right]+\sum_{n=1}^{\infty}\left[K A_{2, n}\right]\right]-k_{a c 2}^{2}\left[K A_{2,1}\right]$

(S.1. 14.1) $m>1: \frac{d\left[K A_{1, m}\right]}{d t}=-k_{a 1}^{1}[D]\left[K A_{1, m}\right]+k_{d 1}^{1}\left[K A_{1, m}^{*}\right]+k_{t}^{1}[K]\left[K A_{1, m}^{*}\right]+$ $k_{t}^{2}\left[K A_{1, m}^{*}\right]+k_{d}\left[K A_{1, m}^{*}\right]\left[\sum_{m=1}^{\infty}\left[D_{1, m}^{*}\right]+\sum_{m=1}^{\infty}\left[K A_{1, m}^{*}\right]+\sum_{m=1}^{\infty}\left[D_{2, m}^{*}\right]+\sum_{m=1}^{\infty}\left[K A_{2, m}^{*}\right]\right]-$ $k_{a c 1}^{1}\left[K A_{1, m}\right] *\left[[I]+\sum_{n=1}^{\infty}\left[K A_{1, n}^{*}\right]+\sum_{n=1}^{\infty}\left[R_{1, n}\right]+\sum_{n=1}^{\infty}\left[K A_{2, n}^{*}\right]+\sum_{n=1}^{\infty}\left[R_{2, n}\right]\right]-$ $2 k_{a c 1}^{1}\left[K A_{1, m}\right]\left[\sum_{n=1}^{\infty}\left[K A_{1, n}\right]+\sum_{n=1}^{\infty}\left[K A_{2, n}\right]\right]+k_{a c 1}^{1}\left(\sum_{i=1}^{m-1}\left[K A_{1, i}\right]\left[K A_{1, m-i}\right]\right)+$ $k_{a c 1}^{1}\left(\sum_{i=1}^{m-1}\left[K A_{2, i}\right]\left[K A_{1, m-i}\right]\right)-k_{a c 1}^{2}\left[K A_{1, m}\right]$

(S.1. 14.2) $m>1: \frac{d\left[K A_{2, m}\right]}{d t}=-k_{a 2}^{1}[D]\left[K A_{2, m}\right]+k_{d 2}^{1}\left[K A_{2, m}^{*}\right]+k_{t}^{1}[K]\left[K A_{2, m}^{*}\right]+$ $k_{t}^{2}\left[K A_{2, m}^{*}\right]+k_{d}\left[K A_{2, m}^{*}\right]\left[\sum_{m=1}^{\infty}\left[D_{1, m}^{*}\right]+\sum_{m=1}^{\infty}\left[K A_{1, m}^{*}\right]+\sum_{m=1}^{\infty}\left[D_{2, m}^{*}\right]+\sum_{m=1}^{\infty}\left[K A_{2, m}^{*}\right]\right]-$ $k_{a c 2}^{1}\left[K A_{2, m}\right] *\left[[I]+\sum_{n=1}^{\infty}\left[K A_{1, n}^{*}\right]+\sum_{n=1}^{\infty}\left[R_{1, n}\right]+\sum_{n=1}^{\infty}\left[K A_{2, n}^{*}\right]+\sum_{n=1}^{\infty}\left[R_{2, n}\right]\right]-$ $2 k_{a c 2}^{1}\left[K A_{2, m}\right]\left[\sum_{n=1}^{\infty}\left[K A_{1, n}\right]+\sum_{n=1}^{\infty}\left[K A_{2, n}\right]\right]+k_{a c 2}^{1}\left(\sum_{i=1}^{m-1}\left[K A_{1, i}\right]\left[K A_{2, m-i}\right]\right)+$ $k_{a c 2}^{1}\left(\sum_{i=1}^{m-1}\left[K A_{2, i}\right]\left[K A_{2, m-i}\right]\right)-k_{a c 2}^{2}\left[K A_{1, m}\right]$

(S.1. 15.1) $n>1: \frac{d\left[C_{1, n}\right]}{d t}=k_{a c 1}^{2}\left[K A_{1, n}\right]$

$\left(\right.$ S.1. 15.2) n > 1: $\frac{d\left[c_{2, n}\right]}{d t}=k_{a c 2}^{2}\left[K A_{2, n}\right]$

(S.1. 16) $\frac{d\left[D^{+}\right]}{d t}=k_{t}^{4}[D][K]$ 


\section{S2. Kine tic rate constant definitions}

(S.2.1.1) $k_{a i}^{2}=$ association between DBU and monomer $i$

(S.2. 1.2) $k_{d i}^{2}=$ dissociation between DBU and monomer $i$

$\left(\boldsymbol{S}\right.$. 2. 2) $k_{p(i, j)}^{1}=$ quasi-anionic propagation of species terminated by monomer $i, D_{i, n}^{*}$ or $K A_{i, n}^{*}$, with monomer $j$

(S.2.3) $k_{i j}=$ initiation (activation) of monomer $j$ by $[D-I]$

(S.2. 4.1) $k_{a i}^{1}=$ association between DBU and an interacting $-\mathrm{OH}$ species containing monomer $i$ adjacent to the $\mathrm{OH}$ end group

(S. 2. 4.2) $k_{d i}^{1}=$ dissociation between DBU and an interacting $-\mathrm{OH}$ species containing monomer $i$ adjacent to the $\mathrm{OH}$ end group

$\left(\boldsymbol{S}\right.$. 2. 5) $k_{t}^{1}=$ poisoning of DBU (production of deactivated DBU, $D^{d}$ ) due to a reaction between $K$ and a growing chain

$\left(\boldsymbol{S}\right.$. 2. 6) $k_{t}^{2}=$ poisoning of DBU (production of $D^{d}$ ) due to a spontaneous proton exchange within a growing chain

(S. 2. 7.1) $k_{t}^{3}=$ conversion of DBU by $K$ to $D^{d}$

$\left(\boldsymbol{S}\right.$. 2. 7.2) $k_{t}^{4}=$ conversion of DBU by $K$ to complexed DBU, $D^{+}$

$\left(\right.$ S. 2. 7.3) $\quad k_{t}^{5}=$ conversion of DBU by $D^{+}$to $D^{d}$

(S. 2. 8) $k_{a c i}^{1}=$ inter-chain acylation of OH-terminated species $\left(I, R_{j, n}, K A_{j, n}\right)$ by $\mathrm{KA}$ species $\left(K A_{i, n}^{*}, K A_{i, n}\right)$

$\left(\boldsymbol{S}\right.$. 2. 9) $k_{a c i}^{2}=$ intra-chain acylation of OH-KA-terminated chain $\left(K A_{i, n}\right)$ resulting in a cyclic chain 
S3. Mechanis ms as sociated with the kinetic balance equations in Section S1

Nucleophilic attack pathway (NAP)

(S.3. 1.1) $D+M_{1} \underset{k_{d 1}^{2}}{\stackrel{k_{a 1}^{2}}{\rightleftharpoons}}\left(D_{1,1} \rightleftharpoons\right) K A_{1,1}$

$\left(\right.$ S.3. 1.2) $D+M_{2} \underset{\boldsymbol{k}_{d 2}^{2}}{\stackrel{\boldsymbol{k}_{a 2}^{2}}{\rightleftharpoons}}\left(D_{2,1} \rightleftharpoons\right) K A_{2,1}$

(S.3. 2.1) $K A_{1,1}+D \underset{\boldsymbol{k}_{d 1}^{1}}{\stackrel{\boldsymbol{k}_{a 1}^{1}}{\perp}} K A_{1,1}^{*}$

$\left(\right.$ S.3. 2.2) $K A_{2,1}+D \underset{\boldsymbol{k}_{d 2}^{1}}{\stackrel{\boldsymbol{k}_{a 2}^{1}}{\rightleftharpoons}} K A_{2,1}^{*}$

(S.3.3.1) $K A_{1, m-1}^{*}+M_{1} \stackrel{k_{p(1,1)}^{1}}{\longrightarrow} K A_{1, m}^{*}$

(S.3. 3.2) $K A_{2, m-1}^{*}+M_{1} \stackrel{k_{p(2,1)}^{1}}{\longrightarrow} K A_{1, m}^{*}$

(S.3. 3.3) $K A_{1, m-1}^{*}+M_{2} \stackrel{k_{p(1,2)}^{1}}{\longrightarrow} K A_{2, m}^{*}$

$\left(\right.$ S.3. 3.4) $K A_{2, m-1}^{*}+M_{2} \stackrel{k_{p(2,1)}^{1}}{\longrightarrow} K A_{2, m}^{*}$

(S.3. 4.1) $K A_{1, m}^{*} \underset{\boldsymbol{k}_{d 1}^{1}}{\stackrel{\boldsymbol{k}_{a 1}^{1}}{\rightrightarrows}} K A_{1, m}+D$

$(\boldsymbol{S} .3 .4 .2) K A_{2, m}^{*} \underset{\boldsymbol{k}_{d 2}^{1}}{\stackrel{\boldsymbol{k}_{a 2}^{1}}{\rightrightarrows}} K A_{2, m}+D$

Activated alcohol pathway (AAP)

$\left(\right.$ S.3.5) $D+I \underset{\boldsymbol{k}_{d}^{2}}{\stackrel{\boldsymbol{k}_{a}^{2}}{\rightleftarrows}}[D-I]$

$\left(\right.$ S.3.6.1) $[D-I]+M_{1} \stackrel{k_{i 1}}{\longrightarrow} D_{1,1}^{*}$

$\left(\right.$ S.3.6.2) $[D-I]+M_{2} \stackrel{k_{i 2}}{\longrightarrow} D_{2,1}^{*}$

$\left(\right.$ S.3. 7.1) $D_{1, n-1}^{*}+M_{1} \stackrel{k_{p(1,1)}^{1}}{\longrightarrow} D_{1, n}^{*}$

$\left(\right.$ S. 3. 7.2) $D_{2, n-1}^{*}+M_{1} \stackrel{k_{p(2,1)}^{1}}{\longrightarrow} D_{1, n}^{*}$

$\left(\right.$ S.3. 7.3) $D_{1, n-1}^{*}+M_{2} \stackrel{k_{p(1,2)}^{1}}{\longrightarrow} D_{2, n}^{*}$

$\left(\right.$ S.3. 7.4) $D_{2, n-1}^{*}+M_{2} \stackrel{k_{p(2,2)}^{1}}{\longrightarrow} D_{2, n}^{*}$

$\left(\right.$ S.3. 8.1) $D_{1, n}^{*} \underset{k_{d 1}^{1}}{\stackrel{k_{a 1}^{1}}{F}} R_{1, n}+D$ 
$\left(\right.$ S.3. 8.2) $D_{2, n}^{*} \underset{\boldsymbol{k}_{d 2}^{1}}{\stackrel{\boldsymbol{k}_{a 2}^{1}}{\leftrightarrows}} R_{2, n}+D$

Inter-chain acylation (combination):

$\left(\right.$ S.3. 9.1) $K A_{1, n}^{*}+I \stackrel{k_{a c 1}^{1}}{\longrightarrow} D_{1, n}^{*}+D$

$(\boldsymbol{S} .3 .9 .2) K A_{2, n}^{*}+I \stackrel{k_{a c 2}^{1}}{\longrightarrow} D_{2, n}^{*}+D$

$\left(\right.$ S.3. 10.1) $K A_{1, n}^{*}+R_{1, m} \stackrel{k_{a c 1}^{1}}{\longrightarrow} D_{1, m+n}^{*}+D$

$\left(\right.$ S.3. 10.2) $K A_{2, n}^{*}+R_{1, m} \stackrel{k_{a c 2}^{1}}{\longrightarrow} D_{2, m+n}^{*}+D$

$\left(\right.$ S.3.10.3) $K A_{1, n}^{*}+R_{2, m} \stackrel{k_{a c 1}^{1}}{\longrightarrow} D_{1, m+n}^{*}+D$

$\left(\right.$ S.3. 10.4) $K A_{2, n}^{*}+R_{2, m} \stackrel{k_{a c 2}^{1}}{\longrightarrow} D_{2, m+n}^{*}+D$

(S.3.11.1) $K A_{1, n}^{*}+K A_{1, m} \stackrel{k_{a c 1}^{1}}{\longrightarrow} K A_{1, m+n}^{*}+D$

(S.3.11.2) $K A_{2, n}^{*}+K A_{1, m} \stackrel{k_{a c 2}^{1}}{\longrightarrow} K A_{2, m+n}^{*}+D$

$\left(\right.$ S.3.11.3) $K A_{1, n}^{*}+K A_{2, m} \stackrel{k_{a c 1}^{1}}{\longrightarrow} K A_{1, m+n}^{*}+D$

$\left(\right.$ S.3.11.4) $K A_{2, n}^{*}+K A_{2, m} \stackrel{k_{a c 2}^{1}}{\longrightarrow} K A_{2, m+n}^{*}+D$

(S.3.12.1) $K A_{1, n}+K A_{1, m} \stackrel{k_{a c 1}^{1}}{\longrightarrow} K A_{1, m+n}+D$

(S.3.12.2) $K A_{2, n}+K A_{1, m} \stackrel{k_{a c 1}^{1}}{\longrightarrow} K A_{1, m+n}+D$

(S.3.12.3) $K A_{1, n}+K A_{2, m} \stackrel{k_{a c 2}^{1}}{\longrightarrow} K A_{2, m+n}+D$

(S.3.12.4) $K A_{2, n}+K A_{2, m} \stackrel{k_{a c 2}^{1}}{\longrightarrow} K A_{2, m+n}+D$

(S.3. 13.1) $K A_{1, n}+I \stackrel{k_{a c 1}^{1}}{\longrightarrow} R_{1, n}+D$

(S.3.13.2) $K A_{2, n}+I \stackrel{k_{a c 2}^{1}}{\longrightarrow} R_{2, n}+D$

$\left(\right.$ S.3. 14.1) $K A_{1, n}+R_{1, m} \stackrel{k_{a c 1}^{1}}{\longrightarrow} R_{1, n+m}+D$

(S.3. 14.2) $K A_{2, n}+R_{1, m} \stackrel{k_{a c 2}^{1}}{\longrightarrow} R_{2, n+m}+D$

$\left(\right.$ S.3. 14.3) $K A_{1, n}+R_{2, m} \stackrel{k_{a c 1}^{1}}{\longrightarrow} R_{1, n+m}+D$

(S.3. 14.4) $K A_{2, n}+R_{2, m} \stackrel{k_{a c 2}^{1}}{\longrightarrow} R_{2, n+m}+D$

Intra-chain acylation (cyclization):

$\left(\right.$ S.3. 15.1) $n>1, \quad K A_{1, n} \stackrel{k_{a c 1}^{2}}{\longrightarrow} C_{1, n}+D$ 
$\left(\right.$ S.3. 15.2) $n>1, \quad K A_{2, n} \stackrel{k_{a c 2}^{2}}{\longrightarrow} C_{2, n}+D$

(S.3. 16.1) $K A_{1,1} \stackrel{k_{a c 1}^{2}}{\longrightarrow} M_{1}+D$

(S.3. 16.2) $K A_{2,1} \stackrel{k_{a c 2}^{2}}{\longrightarrow} M_{2}+D$

\section{DBU Deactivation:}

$\left(\right.$ S.3. 17.1) $D_{1, n}^{*}+K \stackrel{k_{t}^{1}}{\longrightarrow} R_{1, n}+D^{d}$

(S.3.17.2) $D_{2, n}^{*}+K \stackrel{k_{t}^{1}}{\longrightarrow} R_{2, n}+D^{d}$

(S.3. 18.1) $K A_{1, m}^{*}+K \stackrel{k_{t}^{1}}{\longrightarrow} K A_{1, m}+D^{d}$

(S.3.18.2) $K A_{2, m}^{*}+K \stackrel{k_{t}^{1}}{\longrightarrow} K A_{2, m}+D^{d}$

$\left(\right.$ S.3.19.1) $D_{1, n}^{*}+D_{1, m}^{*} \stackrel{k_{d}}{\longrightarrow} R_{1, m}+D_{1, n}^{*}+D^{d}$

$\left(\right.$ S.3. 19.2) $D_{2, n}^{*}+D_{1, m}^{*} \stackrel{k_{d}}{\longrightarrow} R_{1, m}+D_{2, n}^{*}+D^{d}$

$\left(\right.$ S.3. 19.3) $D_{1, n}^{*}+D_{2, m}^{*} \stackrel{k_{d}}{\longrightarrow} R_{2, m}+D_{1, n}^{*}+D^{d}$

$\left(\right.$ S.3. 19.4) $D_{2, n}^{*}+D_{2, m}^{*} \stackrel{k_{d}}{\longrightarrow} R_{2, m}+D_{2, n}^{*}+D^{d}$

(S.3. 20.1) $D_{1, n}^{*}+K A_{1, m}^{*} \stackrel{k_{d}}{\longrightarrow} K A_{1, m}+D_{1, n}^{*}+D^{d}$

(S.3. 20.2) $D_{2, n}^{*}+K A_{1, m}^{*} \stackrel{k_{d}}{\longrightarrow} K A_{1, m}+D_{2, n}^{*}+D^{d}$

$\left(\right.$ S.3. 20.3) $D_{1, n}^{*}+K A_{2, m}^{*} \stackrel{k_{d}}{\longrightarrow} K A_{2, m}+D_{1, n}^{*}+D^{d}$

(S.3. 20.4) $D_{2, n}^{*}+K A_{2, m}^{*} \stackrel{k_{d}}{\longrightarrow} K A_{2, m}+D_{2, n}^{*}+D^{d}$

(S.3.21.1) $K A_{1, n}^{*}+D_{1, m}^{*} \stackrel{k_{d}}{\longrightarrow} R_{1, m}+K A_{1, n}^{*}+D^{d}$

$\left(\right.$ S.3. 21.2) $K A_{2, n}^{*}+D_{1, m}^{*} \stackrel{k_{d}}{\longrightarrow} R_{1, m}+K A_{2, n}^{*}+D^{d}$

$\left(\right.$ S.3. 21.3) $K A_{1, n}^{*}+D_{2, m}^{*} \stackrel{k_{d}}{\longrightarrow} R_{2, m}+K A_{1, n}^{*}+D^{d}$

$\left(\right.$ S.3. 21.4) $K A_{2, n}^{*}+D_{2, m}^{*} \stackrel{k_{d}}{\longrightarrow} R_{2, m}+K A_{2, n}^{*}+D^{d}$

(S.3.22.1) $K A_{1, n}^{*}+K A_{1, m}^{*} \stackrel{k_{d}}{\longrightarrow} K A_{1, m}+K A_{1, n}^{*}+D^{d}$

(S.3. 22.2) $K A_{2, n}^{*}+K A_{1, m}^{*} \stackrel{k_{d}}{\longrightarrow} K A_{1, m}+K A_{2, n}^{*}+D^{d}$

(S.3. 22.3) $K A_{1, n}^{*}+K A_{2, m}^{*} \stackrel{k_{d}}{\longrightarrow} K A_{2, m}+K A_{1, n}^{*}+D^{d}$

$\left(\right.$ S.3. 22.4) $K A_{2, n}^{*}+K A_{2, m}^{*} \stackrel{k_{d}}{\longrightarrow} K A_{2, m}+K A_{2, n}^{*}+D^{d}$

(S.3. 23.1) $D_{1, n}^{*} \stackrel{k_{t}^{2}}{\longrightarrow} R_{1, n}+D^{d}$

$\left(\right.$ S.3. 23.2) $D_{2, n}^{*} \stackrel{k_{t}^{2}}{\longrightarrow} R_{2, n}+D^{d}$

$\left(\right.$ S.3. 24.1) $K A_{1, m}^{*} \stackrel{k_{t}^{2}}{\longrightarrow} K A_{1, m}+D^{d}$

$\left(\right.$ S.3. 24.2) $K A_{2, m}^{*} \stackrel{k_{t}^{2}}{\longrightarrow} K A_{2, m}+D^{d}$ 
(S.3. 25) $D+K \stackrel{k_{t}^{3}}{\longrightarrow} D^{d}$

$\left(\right.$ S.3.26) $D+K \stackrel{k_{t}^{4}}{\longrightarrow} D^{+}+D \stackrel{k_{t}^{5}}{\longrightarrow} D^{+}+D^{d}$ 


\section{S4. Moment Definitions}

(S.4. 1.1) $\left[\kappa_{j}^{1}\right]=\sum_{m=1}^{\infty} m^{j}\left[K A_{1, m}^{*}\right](j=0,1,2)$ for DBU-initiated growing chains with monomer 1 (LA) at the terminal ends

(S.4. 1.2) $\left[\kappa_{j}^{2}\right]=\sum_{m=1}^{\infty} m^{j}\left[K A_{2, m}^{*}\right](j=0,1,2)$ for DBU-initiated growing chains with monomer 2 (GL) at the terminal ends

(S.4. 2.1) $\left[\theta_{j}^{1}\right]=\sum_{m=1}^{\infty} m^{j}\left[K A_{1, m}\right](j=0,1,2)$ for DBU-initiated dormant chains with monomer 1 at the terminal end

(S.4. 2.2) $\left[\theta_{j}^{2}\right]=\sum_{m=1}^{\infty} m^{j}\left[K A_{2, m}\right](j=0,1,2)$ for DBU-initiated dormant chains with monomer 2 at the terminal ends

(S.4. 3. 1) $\left[\lambda_{j}^{1}\right]=\sum_{n=1}^{\infty} n^{j}\left[D_{1, n}^{*}\right](j=0,1,2)$ for alcohol-initiated growing chains with monomer 1 at the terminal ends

(S.4. 3.2) $\left[\lambda_{j}^{2}\right]=\sum_{n=1}^{\infty} n^{j}\left[D_{2, n}^{*}\right](j=0,1,2)$ for alcohol-initiated growing chains with monomer 2 at the terminal ends

(S.4. 4. 1) $\left[\tau_{j}^{1}\right]=\sum_{n=1}^{\infty} n^{j}\left[R_{1, n}\right](j=0,1,2)$ for alcohol-initiated dormant chains with monomer 1 at the terminal ends

(S.4. 4. 1) $\left[\tau_{j}^{2}\right]=\sum_{n=1}^{\infty} n^{j}\left[R_{2, n}\right](j=0,1,2)$ for alcohol-initiated dormant chains with monomer 2 at the terminal ends

(S.4. 5. 1) $\left[\varphi_{j}^{1}\right]=\sum_{n=1}^{\infty} n^{j}\left[C_{1, n}\right](j=0,1,2)$ for cyclic chains formed from linear chains with monomer 1 at the terminal ends

(S.4. 5.2) $\left[\varphi_{j}^{2}\right]=\sum_{n=1}^{\infty} n^{j}\left[C_{2, n}\right](j=0,1,2)$ for cyclic chains formed from linear chains with monomer 2 at the terminal ends 
S5. Kine tic balance equations in terms of moments: The original equations are given in Section S1.

(S. 5. 1) $\frac{d[D]}{d t}=-k_{a}^{1}[D][I]+k_{d}^{1}[[D-I]]-k_{a}^{1}[D]\left[\left[\tau_{0}^{1}\right]+\left[\theta_{0}^{1}\right]+\left[\tau_{0}^{2}\right]+\left[\theta_{0}^{2}\right]\right]+$ $k_{d}^{1}\left[\left[\lambda_{0}^{1}\right]+\left[\kappa_{0}^{1}\right]+\left[\lambda_{0}^{2}\right]+\left[\kappa_{0}^{2}\right]\right]-k_{a 1}^{2}[D]\left[M_{1}\right]-k_{a 2}^{2}[D]\left[M_{2}\right]+k_{d 1}^{2}\left[K A_{1,1}\right]+k_{d 2}^{2}\left[K A_{21}\right]-$ $k_{a 1}^{1}\left[K A_{1,1}\right][D]+k_{d 1}^{1}\left[K A_{1,1}^{*}\right]-k_{a 2}^{1}\left[K A_{2,1}\right][D]+k_{d 2}^{1}\left[K A_{2,1}^{*}\right]-k_{t}^{3}[D][K]+$ $k_{a c 1}^{1}\left[\left[\kappa_{0}^{1}\right]+\left[\theta_{0}^{1}\right]\right] *\left[[I]+\left[\tau_{0}^{1}\right]+\left[\theta_{0}^{1}\right]+\left[\tau_{0}^{2}\right]+\left[\theta_{0}^{2}\right]\right]+k_{a c 1}^{1}\left[K A_{1, m}\right]\left[\left[\kappa_{0}^{1}\right]+\left[\kappa_{0}^{2}\right]\right]+$ $k_{a c 2}^{1}\left[\left[\kappa_{0}^{2}\right]+\left[\theta_{0}^{2}\right]\right] *\left[[I]+\left[\tau_{0}^{1}\right]+\left[\theta_{0}^{1}\right]+\left[\tau_{0}^{2}\right]+\left[\theta_{0}^{2}\right]\right]+k_{a c 2}^{1}\left[K A_{2, m}\right]\left[\left[\kappa_{0}^{1}\right]+\left[\kappa_{0}^{2}\right]\right]+$ $k_{a c 1}^{2}\left[\theta_{0}^{1}\right]+k_{a c 2}^{1}\left[\theta_{0}^{2}\right]-k_{t}^{4}[D][K]-k_{t}^{5}[D]\left[D^{+}\right]$

(S.5. 2.1) $\frac{d\left[M_{1}\right]}{d t}=-k_{i 1}[[D-I]]\left[M_{1}\right]-k_{p(1,1)}^{1}\left[M_{1}\right]\left[\left[\lambda_{0}^{1}\right]+\left[\kappa_{0}^{1}\right]\right]-k_{p(2,1)}^{1}\left[M_{1}\right]\left[\left[\lambda_{0}^{2}\right]+\right.$ $\left.\left[\kappa_{0}^{2}\right]\right]-k_{a 1}^{2}[D]\left[M_{1}\right]+k_{d 1}^{2}\left[K A_{1,1}\right]+k_{a c 1}^{2}\left[K A_{1,1}\right]$

(S.5. 2.2) $\frac{d\left[M_{2}\right]}{d t}=-k_{i 2}[[D-I]]\left[M_{2}\right]-k_{p(1,2)}^{1}\left[M_{2}\right]\left[\left[\lambda_{0}^{1}\right]+\left[\kappa_{0}^{1}\right]\right]-k_{p(2,2)}^{1}\left[M_{2}\right]\left[\left[\lambda_{0}^{2}\right]+\right.$ $\left.\left[\kappa_{0}^{2}\right]\right]-k_{a 2}^{2}[D]\left[M_{2}\right]+k_{d 2}^{2}\left[K A_{2,1}\right]+k_{a c 2}^{2}\left[K A_{2,1}\right]$

(S. 5. 3) $\frac{d[I]}{d t}=-k_{a}^{1}[I][D]+k_{d}^{1}[[D-I]]-k_{a c 1}^{1}[I]\left[\left[\kappa_{0}^{1}\right]+\left[\theta_{0}^{1}\right]\right]-k_{a c 2}^{1}[I]\left[\left[\kappa_{0}^{2}\right]+\left[\theta_{0}^{2}\right]\right]$

$\left(\right.$ S.5.4) $\frac{d[D-I]}{d t}=k_{a}^{1}[I][D]-k_{d}^{1}[[D-I]]-k_{i 1}\left[M_{1}\right][D-I]-k_{i 2}\left[M_{2}\right][D-I]$

$(\boldsymbol{S . 5 . 5 )}) \frac{d\left[D^{d}\right]}{d t}=k_{t}^{1}[K]\left[\left[\lambda_{0}^{1}\right]+\left[\kappa_{0}^{1}\right]\right]+k_{t}^{2}\left[\left[\lambda_{0}^{1}\right]+\left[\kappa_{0}^{1}\right]+\left[\lambda_{0}^{2}\right]+\left[\kappa_{0}^{2}\right]\right]+k_{t}^{3}[K][D]+$ $k_{d}\left[\lambda_{0}^{1}\right]\left[\left[\lambda_{0}^{1}\right]+\left[\kappa_{0}^{1}\right]+\left[\lambda_{0}^{2}\right]+\left[\kappa_{0}^{2}\right]\right]+k_{d}\left[\lambda_{0}^{2}\right]\left[\left[\lambda_{0}^{1}\right]+\left[\kappa_{0}^{1}\right]+\left[\lambda_{0}^{2}\right]+\left[\kappa_{0}^{2}\right]\right]+k_{d}\left[\kappa_{0}^{1}\right]\left[\left[\lambda_{0}^{1}\right]+\right.$ $\left.\left[\kappa_{0}^{1}\right]+\left[\lambda_{0}^{2}\right]+\left[\kappa_{0}^{2}\right]\right]+k_{d}\left[\kappa_{0}^{2}\right]\left[\left[\lambda_{0}^{1}\right]+\left[\kappa_{0}^{1}\right]+\left[\lambda_{0}^{2}\right]+\left[\kappa_{0}^{2}\right]\right]+k_{t}^{5}[D]\left[D^{+}\right]$

(S. 5. 6) $\frac{d[K]}{d t}=-k_{t}^{1}[K]\left[\left[\lambda_{0}^{1}\right]+\left[\kappa_{0}^{1}\right]+\left[\lambda_{0}^{2}\right]+\left[\kappa_{0}^{2}\right]\right]-k_{t}^{3}[K][D]-k_{t}^{4}[D][K]$

$\left(\right.$ S. 5. 7.1) $\frac{d\left[K A_{1,1}^{*}\right]}{d t}=-k_{p(1,1)}^{1}\left[M_{1}\right]\left[K A_{1,1}^{*}\right]-k_{p(1,2)}^{1}\left[M_{2}\right]\left[K A_{1,1}^{*}\right]-k_{t}^{1}\left[K A_{1,1}^{*}\right][K]+$ $2 k_{a 1}^{1}[D]\left[K A_{1,1}\right]-2 k_{d 1}^{1}\left[K A_{1,1}^{*}\right]-k_{t}^{2}\left[K A_{1,1}^{*}\right]-k_{d}\left[K A_{1,1}^{*}\right]\left[\left[\lambda_{0}^{1}\right]+\left[\kappa_{0}^{1}\right]+\left[\lambda_{0}^{2}\right]+\left[\kappa_{0}^{2}\right]\right]-$ $k_{a c 1}^{1}\left[K A_{1,1}^{*}\right]\left[[I]+\left[\tau_{0}^{1}\right]+\left[\theta_{0}^{1}\right]+\left[\tau_{0}^{2}\right]+\left[\theta_{0}^{2}\right]\right]$

(S.5. 7.2) $\frac{d\left[K A_{2,1}^{*}\right]}{d t}=-k_{p(2,1)}^{1}\left[M_{1}\right]\left[K A_{2,1}^{*}\right]-k_{p(2,2)}^{1}\left[M_{2}\right]\left[K A_{2,1}^{*}\right]-k_{t}^{1}\left[K A_{2,1}^{*}\right][K]+$ $2 k_{a 2}^{1}[D]\left[K A_{2,1}\right]-2 k_{d 2}^{1}\left[K A_{2,1}^{*}\right]-k_{t}^{2}\left[K A_{2,1}^{*}\right]-k_{d}\left[K A_{2,1}^{*}\right]\left[\left[\lambda_{0}^{1}\right]+\left[\kappa_{0}^{1}\right]+\left[\lambda_{0}^{2}\right]+\left[\kappa_{0}^{2}\right]\right]-$ $k_{a c 2}^{1}\left[K A_{2,1}^{*}\right]\left[[I]+\left[\tau_{0}^{1}\right]+\left[\theta_{0}^{1}\right]+\left[\tau_{0}^{2}\right]+\left[\theta_{0}^{2}\right]\right]$

(S. 5. 8. 1) $\frac{d\left[\kappa_{0}^{1}\right]}{d t}=k_{a 1}^{1}[D]\left[\theta_{0}^{1}\right]-k_{d 1}^{1}\left[\kappa_{0}^{1}\right]-k_{t}^{1}\left[\kappa_{0}^{1}\right][K]-k_{t}^{2}\left[\kappa_{0}^{1}\right]-k_{d}\left[\kappa_{0}^{1}\right]\left[\left[\lambda_{0}^{1}\right]+\left[\kappa_{0}^{1}\right]+\right.$ $\left.\left[\lambda_{0}^{2}\right]+\left[\kappa_{0}^{2}\right]\right]-k_{a c 1}^{1}\left[\kappa_{0}^{1}\right]\left[[I]+\left[\tau_{0}^{1}\right]+\left[\theta_{0}^{1}\right]+\left[\tau_{0}^{2}\right]+\left[\theta_{0}^{2}\right]\right]+k_{a c 1}^{1}\left[\theta_{0}^{1}\right]\left[\kappa_{0}^{1}\right]+k_{a c 1}^{1}\left[\theta_{0}^{2}\right]\left[\kappa_{0}^{1}\right]$ $\left(\right.$ S. 5. 8.2) $\frac{d\left[\kappa_{0}^{2}\right]}{d t}=k_{a 2}^{1}[D]\left[\theta_{0}^{2}\right]-k_{d 2}^{1}\left[\kappa_{0}^{2}\right]-k_{t}^{1}\left[\kappa_{0}^{2}\right][K]-k_{t}^{2}\left[\kappa_{0}^{2}\right]-k_{d}\left[\kappa_{0}^{2}\right]\left[\left[\lambda_{0}^{1}\right]+\left[\kappa_{0}^{1}\right]+\right.$ $\left.\left[\lambda_{0}^{2}\right]+\left[\kappa_{0}^{2}\right]\right]-k_{a c 2}^{1}\left[\kappa_{0}^{2}\right]\left[[I]+\left[\tau_{0}^{1}\right]+\left[\theta_{0}^{1}\right]+\left[\tau_{0}^{2}\right]+\left[\theta_{0}^{2}\right]\right]+k_{a c 2}^{1}\left[\theta_{0}^{2}\right]\left[\kappa_{0}^{2}\right]+k_{a c 2}^{1}\left[\theta_{0}^{1}\right]\left[\kappa_{0}^{2}\right]$ 
(S.5. 9.1) $\frac{d\left[\kappa_{1}^{1}\right]}{d t}=k_{a 1}^{1}[D]\left[\theta_{1}^{1}\right]-k_{d 1}^{1}\left[\kappa_{1}^{1}\right]-k_{t}^{1}\left[\kappa_{1}^{1}\right][K]+k_{p(1,1)}^{1}\left[M_{1}\right]\left[\kappa_{1}^{1}\right]+$ $k_{p(2,1)}^{1}\left[M_{1}\right]\left[\kappa_{1}^{2}\right]-k_{t}^{2}\left[\kappa_{1}^{1}\right]-k_{d}\left[\kappa_{1}^{1}\right]\left[\left[\lambda_{0}^{1}\right]+\left[\kappa_{0}^{1}\right]+\left[\lambda_{0}^{2}\right]+\left[\kappa_{0}^{2}\right]\right]-k_{a c 1}^{1}\left[\kappa_{1}^{1}\right]\left[[I]+\left[\tau_{0}^{1}\right]+\right.$ $\left.\left[\theta_{0}^{1}\right]+\left[\tau_{0}^{2}\right]+\left[\theta_{0}^{2}\right]\right]+k_{a c 1}^{1}\left(\left[\theta_{1}^{1}\right]\left[\kappa_{0}^{1}\right]+\left[\theta_{0}^{1}\right]\left[\kappa_{1}^{1}\right]\right)+k_{a c 1}^{1}\left(\left[\theta_{1}^{2}\right]\left[\kappa_{0}^{1}\right]+\left[\theta_{0}^{2}\right]\left[\kappa_{1}^{1}\right]\right)$

(S.5. 9.2) $\frac{d\left[\kappa_{1}^{2}\right]}{d t}=k_{a 2}^{1}[D]\left[\theta_{1}^{2}\right]-k_{d 2}^{1}\left[\kappa_{1}^{2}\right]-k_{t}^{1}\left[\kappa_{1}^{2}\right][K]+k_{p(1,2)}^{1}\left[M_{2}\right]\left[\kappa_{1}^{1}\right]+$ $k_{p(2,2)}^{1}\left[M_{2}\right]\left[\kappa_{1}^{2}\right]-k_{t}^{2}\left[\kappa_{1}^{2}\right]-k_{d}\left[\kappa_{1}^{2}\right]\left[\left[\lambda_{0}^{1}\right]+\left[\kappa_{0}^{1}\right]+\left[\lambda_{0}^{2}\right]+\left[\kappa_{0}^{2}\right]\right]-k_{a c 2}^{1}\left[\kappa_{1}^{2}\right]\left[[I]+\left[\tau_{0}^{1}\right]+\right.$ $\left.\left[\theta_{0}^{1}\right]+\left[\tau_{0}^{2}\right]+\left[\theta_{0}^{2}\right]\right]+k_{a c 2}^{1}\left(\left[\theta_{1}^{1}\right]\left[\kappa_{0}^{2}\right]+\left[\theta_{0}^{1}\right]\left[\kappa_{1}^{2}\right]\right)+k_{a c 2}^{1}\left(\left[\theta_{1}^{2}\right]\left[\kappa_{0}^{2}\right]+\left[\theta_{0}^{2}\right]\left[\kappa_{1}^{2}\right]\right)$

(S. 5. 10.1) $\frac{d\left[\kappa_{2}^{1}\right]}{d t}=k_{a 1}^{1}[D]\left[\theta_{2}^{1}\right]-k_{d 1}^{1}\left[\kappa_{2}^{1}\right]-k_{t}^{1}\left[\kappa_{2}^{1}\right][K]+k_{p(1,1)}^{1}\left[M_{1}\right]\left[2\left[\kappa_{2}^{1}\right]+\left[\kappa_{0}^{1}\right]\right]+$ $k_{p(2,1)}^{1}\left[M_{1}\right]\left[2\left[\kappa_{2}^{2}\right]+\left[\kappa_{0}^{2}\right]\right]-k_{t}^{2}\left[\kappa_{2}^{1}\right]-k_{d}\left[\kappa_{2}^{1}\right]\left[\left[\lambda_{0}^{1}\right]+\left[\kappa_{0}^{1}\right]+\left[\lambda_{0}^{2}\right]+\left[\kappa_{0}^{2}\right]\right]-k_{a c 1}^{1}\left[\kappa_{2}^{1}\right][[I]+$ $\left.\left[\tau_{0}^{1}\right]+\left[\theta_{0}^{1}\right]+\left[\tau_{0}^{2}\right]+\left[\theta_{0}^{2}\right]\right]+k_{a c 1}^{1}\left(\left[\theta_{2}^{1}\right]\left[\kappa_{0}^{1}\right]+2\left[\theta_{1}^{1}\right]\left[\kappa_{1}^{1}\right]+\left[\theta_{0}^{1}\right]\left[\kappa_{2}^{1}\right]\right)+k_{a c 1}^{1}\left(\left[\theta_{2}^{2}\right]\left[\kappa_{0}^{1}\right]+\right.$ $\left.2\left[\theta_{1}^{2}\right]\left[\kappa_{1}^{1}\right]+\left[\theta_{0}^{2}\right]\left[\kappa_{2}^{1}\right]\right)$

(S. 5. 10.2) $\frac{d\left[\kappa_{2}^{2}\right]}{d t}=k_{a 2}^{1}[D]\left[\theta_{2}^{2}\right]-k_{d 2}^{1}\left[\kappa_{2}^{2}\right]-k_{t}^{1}\left[\kappa_{2}^{2}\right][K]+k_{p(1,2)}^{1}\left[M_{2}\right]\left[2\left[\kappa_{2}^{1}\right]+\left[\kappa_{0}^{1}\right]\right]+$ $k_{p(2,2)}^{1}\left[M_{2}\right]\left[2\left[\kappa_{2}^{2}\right]+\left[\kappa_{0}^{2}\right]\right]-k_{t}^{2}\left[\kappa_{2}^{2}\right]-k_{d}\left[\kappa_{2}^{2}\right]\left[\left[\lambda_{0}^{1}\right]+\left[\kappa_{0}^{1}\right]+\left[\lambda_{0}^{2}\right]+\left[\kappa_{0}^{2}\right]\right]-k_{a c 2}^{1}\left[\kappa_{2}^{2}\right][[I]+$ $\left.\left[\tau_{0}^{1}\right]+\left[\theta_{0}^{1}\right]+\left[\tau_{0}^{2}\right]+\left[\theta_{0}^{2}\right]\right]+k_{a c 2}^{1}\left(\left[\theta_{2}^{1}\right]\left[\kappa_{0}^{2}\right]+2\left[\theta_{1}^{1}\right]\left[\kappa_{1}^{2}\right]+\left[\theta_{0}^{1}\right]\left[\kappa_{2}^{2}\right]\right)+k_{a c 2}^{1}\left(\left[\theta_{2}^{2}\right]\left[\kappa_{0}^{2}\right]+\right.$ $\left.2\left[\theta_{1}^{2}\right]\left[\kappa_{1}^{2}\right]+\left[\theta_{0}^{2}\right]\left[\kappa_{2}^{2}\right]\right)$

(S. 5. 11.1) $\frac{d\left[K A_{1,1}\right]}{d t}=k_{a 1}^{2}\left[M_{1}\right][D]-k_{d 1}^{2}\left[K A_{1,1}\right]-2 k_{a 1}^{1}[D]\left[K A_{1,1}\right]+2 k_{d 1}^{1}\left[K A_{1,1}^{*}\right]+$ $k_{t}^{1}[K]\left[K A_{1,1}^{*}\right]+k_{t}^{2}\left[K A_{1,1}^{*}\right]+k_{d}\left[K A_{1,1}^{*}\right]\left[\left[\lambda_{0}^{1}\right]+\left[\kappa_{0}^{1}\right]+\left[\lambda_{0}^{2}\right]+\left[\kappa_{0}^{2}\right]\right]-k_{a c 1}^{1}\left[K A_{1,1}\right][[I]+$ $\left.\left[\kappa_{0}^{1}\right]+\left[\tau_{0}^{1}\right]+\left[\kappa_{0}^{2}\right]+\left[\tau_{0}^{2}\right]\right]-2 k_{a c 1}^{1}\left[K A_{1,1}\right]\left[\left[\theta_{0}^{1}\right]+\left[\theta_{0}^{2}\right]\right]-k_{a c 1}^{2}\left[K A_{1,1}\right]$

(S.5. 11.2) $\frac{d\left[K A_{2,1}\right]}{d t}=k_{a 2}^{2}\left[M_{2}\right][D]-k_{d 2}^{2}\left[D_{2,1}\right]-2 k_{a 2}^{1}[D]\left[K A_{2,1}\right]+2 k_{d 2}^{1}\left[K A_{2,1}^{*}\right]+$ $k_{t}^{1}[K]\left[K A_{2,1}^{*}\right]+k_{t}^{2}\left[K A_{2,1}^{*}\right]+k_{d}\left[K A_{2,1}^{*}\right]\left[\left[\lambda_{0}^{1}\right]+\left[\kappa_{0}^{1}\right]+\left[\lambda_{0}^{2}\right]+\left[\kappa_{0}^{2}\right]\right]-k_{a c 2}^{1}\left[K A_{2,1}\right][[I]+$ $\left.\left[\kappa_{0}^{1}\right]+\left[\tau_{0}^{1}\right]+\left[\kappa_{0}^{2}\right]+\left[\tau_{0}^{2}\right]\right]-2 k_{a c 2}^{1}\left[K A_{2,1}\right]\left[\left[\theta_{0}^{1}\right]+\left[\theta_{0}^{2}\right]\right]-k_{a c 2}^{2}\left[K A_{2,1}\right]$

(S.5. 12. 1) $\frac{d\left[\theta_{0}^{1}\right]}{d t}=-k_{a 1}^{1}[D]\left[\theta_{0}^{1}\right]+k_{d 1}^{1}\left[\kappa_{0}^{1}\right]+k_{t}^{1}[K]\left[\kappa_{0}^{1}\right]+k_{t}^{2}\left[\kappa_{0}^{1}\right]+k_{d}\left[\kappa_{0}^{1}\right]\left[\left[\lambda_{0}^{1}\right]+\right.$ $\left.\left[\kappa_{0}^{1}\right]+\left[\lambda_{0}^{2}\right]+\left[\kappa_{0}^{2}\right]\right]-k_{a c 1}^{1}\left[\theta_{0}^{1}\right]\left[[I]+\left[\kappa_{0}^{1}\right]+\left[\tau_{0}^{1}\right]+\left[\kappa_{0}^{2}\right]+\left[\tau_{0}^{2}\right]\right]-2 k_{a c 1}^{1}\left[\theta_{0}^{1}\right]\left[\left[\theta_{0}^{1}\right]+\left[\theta_{0}^{2}\right]\right]+$ $k_{a c 1}^{1}\left[\theta_{0}^{1}\right]\left[\left[\theta_{0}^{1}\right]+\left[\theta_{0}^{2}\right]\right]-k_{a c 1}^{2}\left[\theta_{0}^{1}\right]$

$\left(\right.$ S. 5. 12.2) $\frac{d\left[\theta_{0}^{2}\right]}{d t}=-k_{a 2}^{1}[D]\left[\theta_{0}^{2}\right]+k_{d 2}^{1}\left[\kappa_{0}^{2}\right]+k_{t}^{1}[K]\left[\kappa_{0}^{2}\right]+k_{t}^{2}\left[\kappa_{0}^{2}\right]+k_{d}\left[\kappa_{0}^{2}\right]\left[\left[\lambda_{0}^{1}\right]+\right.$ $\left.\left[\kappa_{0}^{1}\right]+\left[\lambda_{0}^{2}\right]+\left[\kappa_{0}^{2}\right]\right]-k_{a c 2}^{1}\left[\theta_{0}^{2}\right]\left[[I]+\left[\kappa_{0}^{1}\right]+\left[\tau_{0}^{1}\right]+\left[\kappa_{0}^{2}\right]+\left[\tau_{0}^{2}\right]\right]-2 k_{a c 2}^{1}\left[\theta_{0}^{2}\right]\left[\left[\theta_{0}^{1}\right]+\left[\theta_{0}^{2}\right]\right]+$ $k_{a c 2}^{1}\left[\theta_{0}^{2}\right]\left[\left[\theta_{0}^{1}\right]+\left[\theta_{0}^{2}\right]\right]-k_{a c 2}^{2}\left[\theta_{0}^{2}\right]$

(S.5. 13.1) $\frac{d\left[\theta_{1}^{1}\right]}{d t}=-k_{a 1}^{1}[D]\left[\theta_{1}^{1}\right]+k_{d 1}^{1}\left[\kappa_{1}^{1}\right]+k_{t}^{1}[K]\left[\kappa_{1}^{1}\right]+k_{t}^{2}\left[\kappa_{1}^{1}\right]+k_{d}\left[\kappa_{1}^{1}\right]\left[\left[\lambda_{0}^{1}\right]+\right.$ $\left.\left[\kappa_{0}^{1}\right]+\left[\lambda_{0}^{2}\right]+\left[\kappa_{0}^{2}\right]\right]-k_{a c 1}^{1}\left[\theta_{1}^{1}\right]\left[[I]+\left[\kappa_{0}^{1}\right]+\left[\tau_{0}^{1}\right]+\left[\kappa_{0}^{2}\right]+\left[\tau_{0}^{2}\right]\right]-2 k_{a c 1}^{1}\left[\theta_{1}^{1}\right]\left[\left[\theta_{0}^{1}\right]+\left[\theta_{0}^{2}\right]\right]+$ $2 k_{a c 1}^{1}\left[\theta_{1}^{1}\right]\left[\left[\theta_{0}^{1}\right]+\left[\theta_{0}^{2}\right]\right]-k_{a c 1}^{2}\left[\theta_{1}^{1}\right]$ 
(S.5. 13.2) $\frac{d\left[\theta_{1}^{2}\right]}{d t}=-k_{a 2}^{1}[D]\left[\theta_{1}^{2}\right]+k_{d 2}^{1}\left[\kappa_{1}^{2}\right]+k_{t}^{1}[K]\left[\kappa_{1}^{2}\right]+k_{t}^{2}\left[\kappa_{1}^{2}\right]+k_{d}\left[\kappa_{1}^{2}\right]\left[\left[\lambda_{0}^{1}\right]+\right.$ $\left.\left[\kappa_{0}^{1}\right]+\left[\lambda_{0}^{2}\right]+\left[\kappa_{0}^{2}\right]\right]-k_{a c 2}^{1}\left[\theta_{1}^{2}\right]\left[[I]+\left[\kappa_{0}^{1}\right]+\left[\tau_{0}^{1}\right]+\left[\kappa_{0}^{2}\right]+\left[\tau_{0}^{2}\right]\right]-2 k_{a c 2}^{1}\left[\theta_{1}^{2}\right]\left[\left[\theta_{0}^{1}\right]+\left[\theta_{0}^{2}\right]\right]+$ $2 k_{a c 2}^{1}\left[\theta_{1}^{2}\right]\left[\left[\theta_{0}^{1}\right]+\left[\theta_{0}^{2}\right]\right]-k_{a c 2}^{2}\left[\theta_{1}^{2}\right]$

(S.5. 14. 1) $\frac{d\left[\theta_{2}^{1}\right]}{d t}=-k_{a 1}^{1}[D]\left[\theta_{2}^{1}\right]+k_{d 1}^{1}\left[\kappa_{2}^{1}\right]+k_{t}^{1}[K]\left[\kappa_{2}^{1}\right]+k_{t}^{2}\left[\kappa_{2}^{1}\right]+k_{d}\left[\kappa_{2}^{1}\right]\left[\left[\lambda_{0}^{1}\right]+\right.$ $\left.\left[\kappa_{0}^{1}\right]+\left[\lambda_{0}^{2}\right]+\left[\kappa_{0}^{2}\right]\right]-k_{a c 1}^{1}\left[\theta_{2}^{1}\right]\left[[I]+\left[\kappa_{0}^{1}\right]+\left[\tau_{0}^{1}\right]+\left[\kappa_{0}^{2}\right]+\left[\tau_{0}^{2}\right]\right]-2 k_{a c 1}^{1}\left[\theta_{2}^{1}\right]\left[\left[\theta_{0}^{1}\right]+\left[\theta_{0}^{2}\right]\right]+$ $2 k_{a c 1}^{1}\left(\left[\theta_{2}^{1}\right]\left[\theta_{0}^{1}\right]+\left[\theta_{1}^{1}\right]\left[\theta_{1}^{1}\right]\right)+2 k_{a c 1}^{1}\left(\left[\theta_{2}^{1}\right]\left[\theta_{0}^{2}\right]+\left[\theta_{1}^{1}\right]\left[\theta_{1}^{2}\right]\right)-k_{a c 1}^{2}\left[\theta_{2}^{1}\right]$

(S. 5. 14.2) $\frac{d\left[\theta_{2}^{2}\right]}{d t}=-k_{a 2}^{1}[D]\left[\theta_{2}^{2}\right]+k_{d 2}^{1}\left[\kappa_{2}^{2}\right]+k_{t}^{1}[K]\left[\kappa_{2}^{2}\right]+k_{t}^{2}\left[\kappa_{2}^{2}\right]+k_{d}\left[\kappa_{2}^{2}\right]\left[\left[\lambda_{0}^{1}\right]+\right.$ $\left.\left[\kappa_{0}^{1}\right]+\left[\lambda_{0}^{2}\right]+\left[\kappa_{0}^{2}\right]\right]-k_{a c 2}^{1}\left[\theta_{2}^{2}\right]\left[[I]+\left[\kappa_{0}^{1}\right]+\left[\tau_{0}^{1}\right]+\left[\kappa_{0}^{2}\right]+\left[\tau_{0}^{2}\right]\right]-2 k_{a c 2}^{1}\left[\theta_{2}^{2}\right]\left[\left[\theta_{0}^{1}\right]+\left[\theta_{0}^{2}\right]\right]+$ $2 k_{a c 2}^{1}\left(\left[\theta_{2}^{2}\right]\left[\theta_{0}^{1}\right]+\left[\theta_{1}^{2}\right]\left[\theta_{1}^{1}\right]\right)+2 k_{a c 2}^{1}\left(\left[\theta_{2}^{2}\right]\left[\theta_{0}^{2}\right]+\left[\theta_{1}^{2}\right]\left[\theta_{1}^{2}\right]\right)-k_{a c 2}^{2}\left[\theta_{2}^{2}\right]$

(S. 5. 15. 1) $\frac{d\left[D_{1,1}^{*}\right]}{d t}=k_{i 1}\left[M_{1}\right]\left[[D-I]+k_{a 1}^{1}[D]\left[R_{1,1}\right]-k_{d 1}^{1}\left[D_{1,1}^{*}\right]-k_{p(1,1)}^{1}\left[M_{1}\right]\left[D_{1,1}^{*}\right]-\right.$ $k_{p(1,2)}^{1}\left[M_{2}\right]\left[D_{1,1}^{*}\right]-k_{t}^{1}[K]\left[D_{1,1}^{*}\right]-k_{t}^{2}\left[D_{1,1}^{*}\right]-k_{d}\left[D_{1,1}^{*}\right]\left[\left[\lambda_{0}^{1}\right]+\left[k_{0}^{1}\right]+\left[\lambda_{0}^{2}\right]+\left[\kappa_{0}^{2}\right]\right]+$ $k_{a c 1}^{1}[I]\left[K A_{1,1}^{*}\right]$

(S. 5. 15.2) $\frac{d\left[D_{2,1}^{*}\right]}{d t}=k_{i 2}\left[M_{2}\right][[D-I]]+k_{a}^{1}[D]\left[R_{2,1}\right]-k_{d}^{1}\left[D_{2,1}^{*}\right]-k_{p(2,1)}^{1}\left[M_{1}\right]\left[D_{2,1}^{*}\right]-$ $k_{p(2,2)}^{1}\left[M_{2}\right]\left[D_{2,1}^{*}\right]-k_{t}^{1}[K]\left[D_{2,1}^{*}\right]-k_{t}^{2}\left[D_{2,1}^{*}\right]-k_{d}\left[D_{2,1}^{*}\right]\left[\left[\lambda_{0}^{1}\right]+\left[\kappa_{0}^{1}\right]+\left[\lambda_{0}^{2}\right]+\left[\kappa_{0}^{2}\right]\right]+$ $k_{a c 2}^{1}[I]\left[K A_{2,1}^{*}\right]$

(S. 5. 16. 1) $\frac{d\left[\lambda_{0}^{1}\right]}{d t}=k_{i 1}\left[M_{1}\right][[D-I]]+k_{a 1}^{1}[D]\left[\tau_{0}^{1}\right]-k_{d 1}^{1}\left[\lambda_{0}^{1}\right]-k_{t}^{1}[K]\left[\lambda_{0}^{1}\right]-k_{t}^{2}\left[\lambda_{0}^{1}\right]-$ $k_{d}\left[\lambda_{0}^{1}\right]\left[\left[\lambda_{0}^{1}\right]+\left[\kappa_{0}^{1}\right]+\left[\lambda_{0}^{2}\right]+\left[\kappa_{0}^{2}\right]\right]+k_{a c 1}^{1}[I]\left[\kappa_{0}^{1}\right]+k_{a c 1}^{1}\left[\tau_{0}^{1}\right]\left[\kappa_{0}^{1}\right]+k_{a c 1}^{1}\left[\tau_{0}^{1}\right]\left[\kappa_{0}^{2}\right]$

(S.5. 16.2) $\frac{d\left[\lambda_{0}^{2}\right]}{d t}=k_{i 2}\left[M_{2}\right][[D-I]]+k_{a 2}^{1}[D]\left[\tau_{0}^{2}\right]-k_{d 2}^{1}\left[\lambda_{0}^{2}\right]-k_{t}^{1}[K]\left[\lambda_{0}^{2}\right]-k_{t}^{2}\left[\lambda_{0}^{2}\right]-$ $k_{d}\left[\lambda_{0}^{2}\right]\left[\left[\lambda_{0}^{1}\right]+\left[\kappa_{0}^{1}\right]+\left[\lambda_{0}^{2}\right]+\left[\kappa_{0}^{2}\right]\right]+k_{a c 2}^{1}[I]\left[\kappa_{0}^{2}\right]+k_{a c 2}^{1}\left[\tau_{0}^{2}\right]\left[\kappa_{0}^{1}\right]+k_{a c 2}^{1}\left[\tau_{0}^{2}\right]\left[\kappa_{0}^{2}\right]$

(S. 5. 17.1) $\frac{d\left[\lambda_{1}^{1}\right]}{d t}=k_{i 1}\left[M_{1}\right][[D-I]]+k_{a 1}^{1}[D]\left[\tau_{1}^{1}\right]-k_{d 1}^{1}\left[\lambda_{1}^{1}\right]-k_{t}^{1}[K]\left[\lambda_{1}^{1}\right]+$ $k_{p(1,1)}^{1}\left[M_{1}\right]\left[\lambda_{1}^{1}\right]+k_{p(2,1)}^{1}\left[M_{1}\right]\left[\lambda_{1}^{2}\right]-k_{t}^{2}\left[\lambda_{1}^{1}\right]-k_{d}\left[\lambda_{1}^{1}\right]\left[\left[\lambda_{0}^{1}\right]+\left[\kappa_{0}^{1}\right]+\left[\lambda_{0}^{2}\right]+\left[\kappa_{0}^{2}\right]\right]+$ $k_{a c 1}^{1}[I]\left[\kappa_{1}^{1}\right]+k_{a c 1}^{1}\left(\left[\tau_{0}^{1}\right]\left[\kappa_{0}^{1}\right]+\left[\tau_{0}^{1}\right]\left[\kappa_{1}^{1}\right]\right)+k_{a c 1}^{1}\left(\left[\tau_{0}^{1}\right]\left[\kappa_{0}^{2}\right]+\left[\tau_{0}^{1}\right]\left[\kappa_{1}^{2}\right]\right)$

(S. 5. 17.2) $\frac{d\left[\lambda_{1}^{2}\right]}{d t}=k_{i 2}\left[M_{2}\right][[D-I]]+k_{a 2}^{1}[D]\left[\tau_{1}^{2}\right]-k_{d 2}^{1}\left[\lambda_{1}^{2}\right]-k_{t}^{1}[K]\left[\lambda_{1}^{2}\right]+$ $k_{p(1,2)}^{1}\left[M_{2}\right]\left[\lambda_{1}^{1}\right]+k_{p(2,2)}^{1}\left[M_{2}\right]\left[\lambda_{1}^{2}\right]-k_{t}^{2}\left[\lambda_{1}^{2}\right]-k_{d}\left[\lambda_{1}^{2}\right]\left[\left[\lambda_{0}^{1}\right]+\left[\kappa_{0}^{1}\right]+\left[\lambda_{0}^{2}\right]+\left[\kappa_{0}^{2}\right]\right]+$ $k_{a c 2}^{1}[I]\left[\kappa_{1}^{2}\right]+k_{a c 2}^{1}\left(\left[\tau_{0}^{2}\right]\left[\kappa_{0}^{1}\right]+\left[\tau_{0}^{2}\right]\left[\kappa_{1}^{1}\right]\right)+k_{a c 2}^{1}\left(\left[\tau_{0}^{2}\right]\left[\kappa_{0}^{2}\right]+\left[\tau_{0}^{2}\right]\left[\kappa_{1}^{2}\right]\right)$

(S. 5. 18.1) $\frac{d\left[\lambda_{2}^{1}\right]}{d t}=k_{i 1}\left[M_{1}\right][[D-I]]+k_{a 1}^{1}[D]\left[\tau_{2}^{1}\right]-k_{d 1}^{1}\left[\lambda_{2}^{1}\right]-k_{t}^{1}[K]\left[\lambda_{2}^{1}\right]+$ $k_{p(1,1)}^{1}\left[M_{1}\right]\left[2\left[\lambda_{1}^{1}\right]+\left[\lambda_{0}^{1}\right]\right]+k_{p(2,1)}^{1}\left[M_{1}\right]\left[2\left[\lambda_{1}^{2}\right]+\left[\lambda_{0}^{2}\right]\right]-k_{t}^{2}\left[\lambda_{2}^{1}\right]-k_{d}\left[\lambda_{2}^{1}\right]\left[\left[\lambda_{0}^{1}\right]+\left[\kappa_{0}^{1}\right]+\right.$ $\left.\left[\lambda_{0}^{2}\right]+\left[\kappa_{0}^{2}\right]\right]+k_{a c 1}^{1}[I]\left[\kappa_{2}^{1}\right]+k_{a c 1}^{1}\left(\left[\tau_{2}^{1}\right]\left[\kappa_{0}^{1}\right]+2\left[\tau_{1}^{1}\right]\left[\kappa_{1}^{1}\right]+\left[\tau_{0}^{1}\right]\left[\kappa_{2}^{1}\right]\right)+k_{a c 1}^{1}\left(\left[\tau_{2}^{1}\right]\left[\kappa_{0}^{2}\right]+\right.$ $\left.2\left[\tau_{1}^{1}\right]\left[\kappa_{1}^{2}\right]+\left[\tau_{0}^{1}\right]\left[\kappa_{2}^{2}\right]\right)$ 
(S.5. 18.2) $\frac{d\left[\lambda_{2}^{2}\right]}{d t}=k_{i 2}\left[M_{2}\right][[D-I]]+k_{a 2}^{1}[D]\left[\tau_{2}^{2}\right]-k_{d 2}^{1}\left[\lambda_{2}^{2}\right]-k_{t}^{1}[K]\left[\lambda_{2}^{2}\right]+$ $k_{p(1,2)}^{1}\left[M_{2}\right]\left[2\left[\lambda_{1}^{1}\right]+\left[\lambda_{0}^{1}\right]\right]+k_{p(2,2)}^{1}\left[M_{2}\right]\left[2\left[\lambda_{1}^{2}\right]+\left[\lambda_{0}^{2}\right]\right]-k_{t}^{2}\left[\lambda_{2}^{2}\right]-k_{d}\left[\lambda_{2}^{2}\right]\left[\left[\lambda_{0}^{1}\right]+\left[\kappa_{0}^{1}\right]+\right.$ $\left.\left[\lambda_{0}^{2}\right]+\left[\kappa_{0}^{2}\right]\right]+k_{a c 2}^{1}[I]\left[\kappa_{2}^{2}\right]+k_{a c 2}^{1}\left(\left[\tau_{2}^{2}\right]\left[\kappa_{0}^{1}\right]+2\left[\tau_{1}^{2}\right]\left[\kappa_{1}^{1}\right]+\left[\tau_{0}^{2}\right]\left[\kappa_{2}^{1}\right]\right)+k_{a c 2}^{1}\left(\left[\tau_{2}^{2}\right]\left[\kappa_{0}^{2}\right]+\right.$ $\left.2\left[\tau_{1}^{2}\right]\left[\kappa_{1}^{2}\right]+\left[\tau_{0}^{2}\right]\left[\kappa_{2}^{2}\right]\right)$

(S. 5. 19.1) $\frac{d\left[R_{1,1}\right]}{d t}=-k_{a 1}^{1}[D]\left[R_{1,1}\right]+k_{d 1}^{1}\left[D_{1,1}^{*}\right]+k_{t}^{1}[K]\left[D_{1,1}^{*}\right]+k_{t}^{2}\left[D_{1,1}^{*}\right]+$ $k_{d}\left[D_{1,1}^{*}\right]\left[\left[\lambda_{0}^{1}\right]+\left[\kappa_{0}^{1}\right]+\left[\lambda_{0}^{2}\right]+\left[\kappa_{0}^{2}\right]\right]+k_{a c 1}^{1}[I]\left[K A_{1,1}\right]-k_{a c 1}^{1}\left[R_{1,1}\right]\left[\left[\left[\theta_{0}^{1}\right]+\left[\theta_{0}^{2}\right]+\left[\kappa_{0}^{1}\right]+\right.\right.$ $\left.\left.\left[\kappa_{0}^{2}\right]\right]\right]$

(S. 5. 19.2) $\frac{d\left[R_{2,1}\right]}{d t}=-k_{a 2}^{1}[D]\left[R_{2,1}\right]+k_{d 2}^{1}\left[D_{2,1}^{*}\right]+k_{t}^{1}[K]\left[D_{2,1}^{*}\right]+k_{t}^{2}\left[D_{2,1}^{*}\right]+$ $k_{d}\left[D_{2,1}^{*}\right]\left[\left[\lambda_{0}^{1}\right]+\left[\kappa_{0}^{1}\right]+\left[\lambda_{0}^{2}\right]+\left[\kappa_{0}^{2}\right]\right]+k_{a c 2}^{1}[I]\left[K A_{2,1}\right]-k_{a c 2}^{1}\left[R_{2,1}\right]\left[\left[\left[\theta_{0}^{1}\right]+\left[\theta_{0}^{2}\right]+\left[\kappa_{0}^{1}\right]+\right.\right.$ $\left.\left.\left[\kappa_{0}^{2}\right]\right]\right]$

(S.5. 20.1) $\frac{d\left[\tau_{0}^{1}\right]}{d t}=-k_{a 1}^{1}[D]\left[\tau_{0}^{1}\right]+k_{d 1}^{1}\left[\lambda_{0}^{1}\right]+k_{t}^{1}[K]\left[\lambda_{0}^{1}\right]+k_{t}^{2}\left[\lambda_{0}^{1}\right]+k_{d}\left[\lambda_{0}^{1}\right]\left[\left[\lambda_{0}^{1}\right]+\left[\kappa_{0}^{1}\right]+\right.$ $\left.\left[\lambda_{0}^{2}\right]+\left[\kappa_{0}^{2}\right]\right]+k_{a c 1}^{1}[I]\left[\theta_{0}^{1}\right]-k_{a c 1}^{1}\left[\tau_{0}^{1}\right]\left[\left[\theta_{0}^{1}\right]+\left[\theta_{0}^{2}\right]+\left[\kappa_{0}^{1}\right]+\left[\kappa_{0}^{2}\right]\right]+k_{a c 1}^{1}\left[\tau_{0}^{1}\right]\left[\theta_{0}^{1}\right]$ $+k_{a c 1}^{1}\left[\tau_{0}^{2}\right]\left[\theta_{0}^{1}\right]$

(S.5. 20.2) $\frac{d\left[\tau_{0}^{2}\right]}{d t}=-k_{a 2}^{1}[D]\left[\tau_{0}^{2}\right]+k_{d 2}^{1}\left[\lambda_{0}^{2}\right]+k_{t}^{1}[K]\left[\lambda_{0}^{2}\right]+k_{t}^{2}\left[\lambda_{0}^{2}\right]+k_{d}\left[\lambda_{0}^{2}\right]\left[\left[\lambda_{0}^{1}\right]+\left[\kappa_{0}^{1}\right]+\right.$ $\left.\left[\lambda_{0}^{2}\right]+\left[\kappa_{0}^{2}\right]\right]+k_{a c 2}^{1}[I]\left[\theta_{0}^{2}\right]-k_{a c 2}^{1}\left[\tau_{0}^{2}\right]\left[\left[\theta_{0}^{1}\right]+\left[\theta_{0}^{2}\right]+\left[\kappa_{0}^{1}\right]+\left[\kappa_{0}^{2}\right]\right]+k_{a c 2}^{1}\left[\tau_{0}^{1}\right]\left[\theta_{0}^{2}\right]$ $+k_{a c 2}^{1}\left[\tau_{0}^{2}\right]\left[\theta_{0}^{2}\right]$

(S.5. 21.1) $\frac{d\left[\tau_{1}^{1}\right]}{d t}=-k_{a 1}^{1}[D]\left[\tau_{1}^{1}\right]+k_{d 1}^{1}\left[\lambda_{1}^{1}\right]+k_{t}^{1}[K]\left[\lambda_{1}^{1}\right]+k_{t}^{2}\left[\lambda_{1}^{1}\right]+k_{d}\left[\lambda_{1}^{1}\right]\left[\left[\lambda_{0}^{1}\right]+\left[\kappa_{0}^{1}\right]+\right.$ $\left.\left[\lambda_{0}^{2}\right]+\left[\kappa_{0}^{2}\right]\right]+k_{a c 1}^{1}[I]\left[\theta_{1}^{1}\right]-k_{a c 1}^{1}\left[\tau_{1}^{1}\right]\left[\left[\theta_{0}^{1}\right]+\left[\theta_{0}^{2}\right]+\left[\kappa_{0}^{1}\right]+\left[\kappa_{0}^{2}\right]\right]+k_{a c 1}^{1}\left(\left[\tau_{1}^{1}\right]\left[\theta_{0}^{1}\right]+\right.$ $\left.\left[\tau_{0}^{1}\right]\left[\theta_{1}^{1}\right]\right)+k_{a c 1}^{1}\left(\left[\tau_{1}^{2}\right]\left[\theta_{0}^{1}\right]+\left[\tau_{0}^{2}\right]\left[\theta_{1}^{1}\right]\right)$

(S.5. 21.2) $\frac{d\left[\tau_{1}^{2}\right]}{d t}=-k_{a 2}^{1}[D]\left[\tau_{1}^{2}\right]+k_{d 2}^{1}\left[\lambda_{1}^{2}\right]+k_{t}^{1}[K]\left[\lambda_{1}^{2}\right]+k_{t}^{2}\left[\lambda_{1}^{2}\right]+k_{d}\left[\lambda_{1}^{2}\right]\left[\left[\lambda_{0}^{1}\right]+\left[\kappa_{0}^{1}\right]+\right.$ $\left.\left[\lambda_{0}^{2}\right]+\left[\kappa_{0}^{2}\right]\right]+k_{a c 2}^{1}[I]\left[\theta_{1}^{2}\right]-k_{a c 2}^{1}\left[\tau_{1}^{2}\right]\left[\left[\theta_{0}^{1}\right]+\left[\theta_{0}^{2}\right]+\left[\kappa_{0}^{1}\right]+\left[\kappa_{0}^{2}\right]\right]+k_{a c 2}^{1}\left(\left[\tau_{1}^{1}\right]\left[\theta_{0}^{2}\right]+\right.$ $\left.\left[\tau_{0}^{1}\right]\left[\theta_{1}^{2}\right]\right)+k_{a c 2}^{1}\left(\left[\tau_{1}^{2}\right]\left[\theta_{0}^{2}\right]+\left[\tau_{0}^{2}\right]\left[\theta_{1}^{2}\right]\right)$

$\left(\right.$ S.5. 22.1) $\frac{d\left[\tau_{2}^{1}\right]}{d t}=-k_{a 1}^{1}[D]\left[\tau_{2}^{1}\right]+k_{d 1}^{1}\left[\lambda_{2}^{1}\right]+k_{t}^{1}[K]\left[\lambda_{2}^{1}\right]+k_{t}^{2}\left[\lambda_{2}^{1}\right]+k_{d}\left[\lambda_{2}^{1}\right]\left[\left[\lambda_{0}^{1}\right]+\left[\kappa_{0}^{1}\right]+\right.$ $\left.\left[\lambda_{0}^{2}\right]+\left[\kappa_{0}^{2}\right]\right]+k_{a c 1}^{1}[I]\left[\theta_{2}^{1}\right]-k_{a c 1}^{1}\left[\tau_{2}^{1}\right]\left[\left[\theta_{0}^{1}\right]+\left[\theta_{0}^{2}\right]+\left[\kappa_{0}^{1}\right]+\left[\kappa_{0}^{2}\right]\right]+k_{a c 1}^{1}\left(\left[\tau_{2}^{1}\right]\left[\theta_{0}^{1}\right]+\right.$ $\left.2\left[\tau_{1}^{1}\right]\left[\theta_{1}^{1}\right]+\left[\tau_{0}^{1}\right]\left[\theta_{2}^{1}\right]\right)+k_{a c 1}^{1}\left(\left[\tau_{2}^{2}\right]\left[\theta_{0}^{1}\right]+2\left[\tau_{1}^{2}\right]\left[\theta_{1}^{1}\right]+\left[\tau_{0}^{2}\right]\left[\theta_{2}^{1}\right]\right)$

$\left(\right.$ S.5. 22.2) $\frac{d\left[\tau_{2}^{2}\right]}{d t}=-k_{a 2}^{1}[D]\left[\tau_{2}^{2}\right]+k_{d 2}^{1}\left[\lambda_{2}^{2}\right]+k_{t}^{1}[K]\left[\lambda_{2}^{2}\right]+k_{t}^{2}\left[\lambda_{2}^{2}\right]+k_{d}\left[\lambda_{2}^{2}\right]\left[\left[\lambda_{0}^{1}\right]+\left[\kappa_{0}^{1}\right]+\right.$ $\left.\left[\lambda_{0}^{2}\right]+\left[\kappa_{0}^{2}\right]\right]+k_{a c 2}^{1}[I]\left[\theta_{2}^{2}\right]-k_{a c 2}^{1}\left[\tau_{2}^{2}\right]\left[\left[\theta_{0}^{1}\right]+\left[\theta_{0}^{2}\right]+\left[\kappa_{0}^{1}\right]+\left[\kappa_{0}^{2}\right]\right]+k_{a c 2}^{1}\left(\left[\tau_{2}^{1}\right]\left[\theta_{0}^{2}\right]+\right.$ $\left.2\left[\tau_{1}^{1}\right]\left[\theta_{1}^{2}\right]+\left[\tau_{0}^{1}\right]\left[\theta_{2}^{2}\right]\right)+k_{a c 2}^{1}\left(\left[\tau_{2}^{2}\right]\left[\theta_{0}^{2}\right]+2\left[\tau_{1}^{2}\right]\left[\theta_{1}^{2}\right]+\left[\tau_{0}^{2}\right]\left[\theta_{2}^{2}\right]\right)$

(S. 5. 23.1) $\frac{d\left[C_{1,1}\right]}{d t}=\frac{d\left[M_{1}\right]}{d t}$ 
$\left(\right.$ S.5. 23.2) $\frac{d\left[C_{2,1}\right]}{d t}=\frac{d\left[M_{2}\right]}{d t}$

(S.5. 24.1) $\frac{d\left[\varphi_{0}^{1}\right]}{d t}=k_{a c 1}^{2}\left(\left[\theta_{0}^{1}\right]-\left[K A_{1,1}\right]\right)$

$(\boldsymbol{S} .5 .24 .2) \frac{d\left[\varphi_{0}^{2}\right]}{d t}=k_{a c 2}^{2}\left(\left[\theta_{0}^{2}\right]-\left[K A_{2,1}\right]\right)$

(S. 5. 25. 1) $\frac{d\left[\varphi_{1}^{1}\right]}{d t}=k_{a c 1}^{2}\left(\left[\theta_{1}^{1}\right]-\left[K A_{1,1}\right]\right)$

$\left(\right.$ S. 5. 25.2) $\frac{d\left[\varphi_{1}^{2}\right]}{d t}=k_{a c 2}^{2}\left(\left[\theta_{1}^{2}\right]-\left[K A_{2,1}\right]\right)$

(S.5. 26.1) $\frac{d\left[\varphi_{2}^{1}\right]}{d t}=k_{a c 1}^{2}\left(\left[\theta_{2}^{1}\right]-\left[K A_{1,1}\right]\right)$

$(\boldsymbol{S . 5 . 2 6 . 2 )}) \frac{d\left[\varphi_{2}^{2}\right]}{d t}=k_{a c 2}^{2}\left(\left[\theta_{2}^{2}\right]-\left[K A_{2,1}\right]\right)$

$\left(\right.$ S.5. 27) $\frac{d\left[D^{+}\right]}{d t}=k_{t}^{4}[D][K]$ 


\section{S6. Calculation of repeat unit sequence lengths from cumulative relative molar concentrations of repeat unit dyads de te rmined by ${ }^{13} \mathrm{C}$ NMR}

Within the terminal model, the normalized instantaneous probability of finding $k$ units of monomer $i$ is given by ${ }^{1}$

$$
S_{i, k}^{\text {inst }}=P_{i i}^{k-1}\left(1-P_{i i}\right)
$$

where $P_{i j}$ is the probability of adding monomer $j$ to a chain end containing monomer $i(i, j=$ 1 (LA) or 2 (GL)). The instantaneous relative molar concentrations of lactate-lactate-lactate, lactate-lactate-glycolate (or glycolate-lactate-lactate), glycolate-glycolate-lactate (or lactateglycolate-glycolate), and glycolate-glycolate-glycolate triads $\left(I_{111}^{\text {inst }}, I_{112}^{\text {inst }}\left(=I_{211}^{\text {inst }}\right), I_{221}^{\text {inst }}\right.$ (= $\left.I_{122}^{\text {inst }}\right)$, and $I_{222}^{\text {inst }}$, respectively) are related to these instantaneous sequence probabilities by

$$
\begin{aligned}
& I_{111}^{\text {inst }} \propto\left(0 \cdot S_{1,1}^{\text {inst }}+2 \cdot S_{1,2}^{\text {inst }}+4 \cdot S_{1,3}^{\text {inst }}+\cdots\right) \\
& I_{112}^{\text {inst }}\left(=I_{211}^{\text {inst }}\right) \propto\left(2 \cdot S_{1,1}^{\text {inst }}+2 \cdot S_{1,2}^{\text {inst }}+2 \cdot S_{1,3}^{\text {inst }}+\cdots\right) \\
& I_{221}^{\text {inst }}\left(=I_{122}^{\text {inst }}\right) \propto\left(2 \cdot S_{2,1}^{\text {inst }}+2 \cdot S_{2,2}^{\text {inst }}+2 \cdot S_{2,3}^{\text {inst }}+\cdots\right) \\
& I_{222}^{\text {inst }} \propto\left(0 \cdot S_{2,1}^{\text {inst }}+2 \cdot S_{2,2}^{\text {inst }}+4 \cdot S_{2,3}^{\text {inst }}+\cdots\right) .
\end{aligned}
$$

Substitution of Eq. (S1) into Eqs. (S2) - (S5) gives

$$
\begin{aligned}
& I_{111}^{\text {inst }}=(\text { const }) \sum_{k=1}^{\infty}(2 k-2) P_{11}^{k-1}\left(1-P_{11}\right)=2(\text { const }) \frac{P_{11}}{1-P_{11}} \\
& I_{112}^{\text {inst }}\left(=I_{211}^{\text {inst }}\right)=2(\text { const }) \sum_{k=1}^{\infty} P_{11}^{k-1}\left(1-P_{11}\right)=2(\text { const }) \\
& I_{221}^{\text {inst }}\left(=I_{122}^{\text {inst }}\right)=2(\text { const }) \sum_{k=1}^{\infty} P_{22}^{k-1}\left(1-P_{22}\right)=2 \text { (const) } \\
& I_{222}^{\text {inst }}=(\text { const }) \sum_{k=1}^{\infty}(2 k-1) P_{22}^{k-1}\left(1-P_{22}\right)=2 \text { (const) } \frac{P_{22}}{1-P_{22}} .
\end{aligned}
$$

From Eqs. (S6) and (S7), one obtains

$$
P_{11}=\frac{I_{111}^{\text {inst }}}{I_{111}^{\text {inst }}+I_{112}^{\text {inst }}}
$$

From Eqs. (S8) and (S9), one obtains

$$
P_{22}=\frac{I_{222}^{\text {inst }}}{I_{222}^{\text {inst }}+I_{221}^{\text {inst }}} \text {. }
$$

Therefore, using Eqs. (3) and (4) of the main text, the instantaneous number- and weightaverage repeat unit sequence lengths can be calculated as

$$
\begin{aligned}
& \left(n_{1}\right)_{n}^{\text {inst }}=2\left(N_{1}\right)_{n}^{\text {inst }}=\frac{2}{1-P_{11}}=2 \frac{I_{11}^{\text {inst }}}{I_{112}^{\text {inst }}}+2 \\
& \left(n_{2}\right)_{n}^{\text {inst }}=2\left(N_{2}\right)_{n}^{i n s t}=\frac{2}{1-P_{22}}=2 \frac{I_{222}^{\text {inst }}}{I_{221}^{\text {inst }}}+2 \\
& \left(n_{1}\right)_{w}^{\text {inst }}=2\left(N_{1}\right)_{w}^{\text {inst }}=2 \frac{1+P_{11}}{1-P_{11}}=4 \frac{I_{111}^{\text {inst }}}{I_{112}^{\text {inst }}}+2 \\
& \left(n_{2}\right)_{w}^{\text {inst }}=2\left(N_{2}\right)_{w}^{\text {inst }}=2 \frac{1+P_{22}}{1-P_{22}}=4 \frac{I_{222}^{\text {inst }}}{I_{221}^{\text {inst }}}+2 .
\end{aligned}
$$

Note the lactate (or glycolate) repeat unit sequence length (" $n_{i}$ ") is twice the LA (or GL) monomer sequence length (" $N_{i}$ "), because when polymerized, each LA (or GL) monomer turns into two lactate (or glycolate) repeat units.

Typically, ${ }^{13} \mathrm{C}$ NMR measurements are performed on final products of polymerization, which give data for the cumulative (instead of instantaneous) relative dyad concentrations $\left(I_{111}^{\text {cumu }}, I_{112}^{\text {cumu }}\left(=I_{211}^{\text {cumu }}\right), I_{221}^{\text {cumu }}\left(=I_{122}^{\text {cumu }}\right)\right.$, and $\left.I_{222}^{\text {cumu }}\right)$. The cumulative number- and weight-average repeat unit sequence lengths can be calculated from the NMR results using the following pre-averaging approximations (analogous to Eqs. (S12) - (S15)): 


$$
\begin{aligned}
& \left(n_{1}\right)_{n}^{\text {cumu }}=2\left(N_{1}\right)_{n}^{\text {cumu }} \cong 2 \frac{I_{111}^{\text {cumu }}}{I_{12}^{\text {cumu }}}+2 \\
& \left(n_{2}\right)_{n}^{\text {cumu }}=2\left(N_{2}\right)_{n}^{\text {cumu }} \cong 2 \frac{I_{222}^{\text {cumu }}}{I_{221}^{\text {cumu }}}+2 \\
& \left(n_{1}\right)_{w}^{\text {cumu }}=2\left(N_{1}\right)_{W}^{\text {cumu }} \cong 4 \frac{I_{111}^{\text {cumu }}}{I_{112}^{\text {cumu }}}+2 \\
& \left(n_{2}\right)_{w}^{\text {cumu }}=2\left(N_{2}\right)_{w}^{\text {cumu }} \cong 4 \frac{I_{\frac{122}{\text { cum }}}^{\text {cum }}}{I_{221}^{\text {cumu }}}+2 .
\end{aligned}
$$

Eqs. (S16) and (S17) are given, respectively, as Eqs. (1) and (2) in the main text. In the PLGA literature, the following simplified (dyadic) notations are commonly used for ${ }^{13} \mathrm{C}$ NMR peak assignments: $I_{111}^{\text {cumu }} \rightarrow$ "I $I_{11} ", I_{112}^{\text {cumu }}\left(=I_{211}^{\text {cumu }}\right) \rightarrow$ "I $I_{12} ", I_{221}^{\text {cumu }}\left(=I_{122}^{\text {cumu }}\right) \rightarrow$ "I $I_{21}$ ", and $I_{222}^{c u m u} \rightarrow$ "I $I_{22}$ ". 


\section{References for the SI}

[1] N. A. Dotson, R. Galván, R. L. Laurence, M. Tirrell, Polymerization Process Modeling; VCH: New York, 1996. 